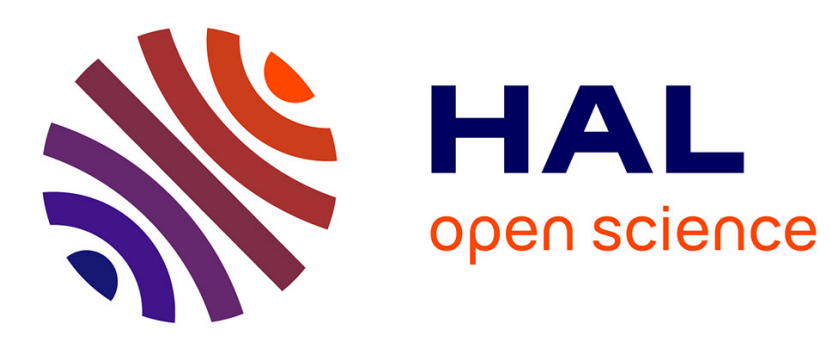

\title{
Fluid-Structure Interactions and Unsteady Kinematics of a Low-Reynolds-Number Rotor
}

\author{
Antonio Alguacil, Thierry Jardin, Nicolas Gourdain
}

\section{To cite this version:}

Antonio Alguacil, Thierry Jardin, Nicolas Gourdain. Fluid-Structure Interactions and Unsteady Kinematics of a Low-Reynolds-Number Rotor. AIAA Journal, 2020, 58 (2), pp.955-967. 10.2514/1.J058230 . hal-03079732

\section{HAL Id: hal-03079732 \\ https://hal.science/hal-03079732}

Submitted on 17 Dec 2020

HAL is a multi-disciplinary open access archive for the deposit and dissemination of scientific research documents, whether they are published or not. The documents may come from teaching and research institutions in France or abroad, or from public or private research centers.
L'archive ouverte pluridisciplinaire HAL, est destinée au dépôt et à la diffusion de documents scientifiques de niveau recherche, publiés ou non, émanant des établissements d'enseignement et de recherche français ou étrangers, des laboratoires publics ou privés. 


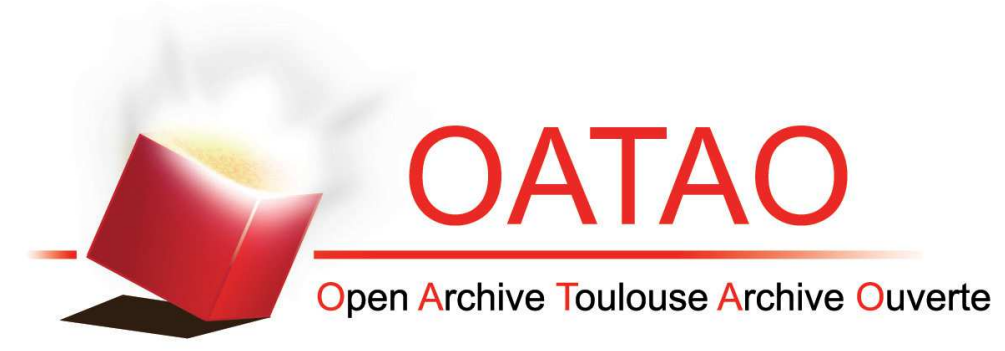

\section{Open Archive Toulouse Archive Ouverte (OATAO)}

OATAO is an open access repository that collects the work of some Toulouse researchers and makes it freely available over the web where possible.

This is an author's version published in: https://oatao.univ-toulouse.fr/27059

Official URL:https://doi.org/10.2514/1.J058230

\section{To cite this version :}

Alguacil, Antonio and Jardin, Thierry and Gourdain, Nicolas Fluid-Structure Interactions and Unsteady Kinematics of a Low-Reynolds-Number Rotor. (2020) AIAA Journal, 58 (2). 955-967. ISSN 0001-1452

Any correspondence concerning this service should be sent to the repository administrator: tech-oatao@listes-diff.inp-toulouse.fr 


\title{
Fluid-Structure Interactions and Unsteady Kinematics of a Low-Reynolds-Number Rotor
}

\author{
Antonio Alguacil,,$*$ Thierry Jardin,,- and Nicolas Gourdain $\stackrel{ \pm}{ \pm}$ \\ ISAE-Supaero, University of Toulouse, 31055 Toulouse, France
}

DOI: $10.2514 / 1 . J 058230$

\begin{abstract}
Micro air vehicles are used for both civil and military applications, like rescue or surveillance. The aerodynamic performance of the rotor is known to be lower than for classical large rotors, due to increased drag at low Reynolds numbers. However, the rotor performance can be improved by taking advantage of the flow unsteadiness and considering unsteady rotor kinematics, like a periodic variation of the rotor pitch. To study such behaviors, it is necessary to develop numerical methods adapted to these fluid-structure interaction phenomena, which are the main objectives of this paper. The method relies on the implementation of fluid-structure interaction capabilities in a lattice-Boltzmann flow solver, which is implemented in a monolithic fashion using generalized coordinates. The validation is first conducted on a vortex-induced vibration test case. Then, numerical simulations are performed for a rotor test case 1) with a forced motion and 2) by coupling the flow with the equation of the dynamics. Some semianalytical models are derived and validated against the numerical simulations to predict the effects of pitching, flapping, and surging on the thrust. The results show that flapping and surging significantly increase the rotor thrust, but at the price of a penalty on the power consumption.
\end{abstract}

\section{Nomenclature}

$C \quad=$ blade chord, $\mathrm{m}$

$C_{p} \quad=$ pressure coefficient

$C_{Q}=$ torque coefficient

$C_{T}=$ thrust coefficient

$D=$ rotor diameter

$K=$ blade stiffness, $\mathrm{N} \cdot \mathrm{m}^{-1}$

$I=$ moment of inertia, $\mathrm{kg} \cdot \mathrm{m}^{2}$

$m \quad=$ mass, $\mathrm{kg}$

$q, \dot{q}=$ generalized coordinate and velocity, $\mathrm{rad}$ and $\mathrm{rad} \cdot \mathrm{s}^{-1}$

$R=$ radius at the rotor tip, $\mathrm{m}$

$R_{S} \quad=$ rotor span, $\mathrm{m}$

$r=$ radius at root, $\mathrm{m}$

$\bar{r} \quad=$ mean rotor radius, $\mathrm{m}$

$U^{*} \quad=$ reduced velocity; Eq. (5)

$V_{i}=$ induced velocity, $\mathrm{m} \cdot \mathrm{s}^{-1}$

$\alpha, \dot{\alpha}=$ pitching angle and velocity, $\mathrm{rad}$ and $\mathrm{rad} \cdot \mathrm{s}^{-1}$

$\beta, \dot{\beta}=$ flapping angle and velocity, $\operatorname{rad}$ and $\mathrm{rad} \cdot \mathrm{s}^{-1}$

$\Omega=$ rotor rotation speed, $\mathrm{rad} \cdot \mathrm{s}^{-1}$

$\omega, \dot{\omega}=$ surging angle and velocity, $\mathrm{rad}$ and $\mathrm{rad} \cdot \mathrm{s}^{-1}$

$\omega_{m} \quad=$ pulsation of the motion, $\mathrm{rad} \cdot \mathrm{s}^{-1}$

\section{Introduction}

$\mathbf{T}$ HE thrust and torque are parameters of paramount importance when designing a rotor, both for payload and efficiency. Usually, the thrust and torque coefficients are estimated for a rotor as a function of the blade incidence angle $\alpha$ and the rotation speed $\Omega$ such that $C_{T}=k \cdot f(\alpha, \Omega)$ with $k$ a coefficient that depends on the considered geometry [for example, $k=2 \pi$ in the case of a twodimensional (2-D) thin airfoil]. Major efforts have been made recently to improve the performance of rotors by optimizing the

\footnotetext{
*Ph.D. Student, Department of Aerodynamics, Energetics and Propulsion; antonio.alguacil-cabrerizo@isae.fr.

${ }^{\dagger}$ Research Engineer, Department of Aerodynamics, Energetics and Propulsion; thierry.jardin@isae-supaero.fr.

FProfessor, Department of Aerodynamics, Energetics and Propulsion; nicolas.gourdain@isae-supaero.fr.
}

design of blades under steady flow conditions. However, the possibility to increase the rotor performance by taking advantage of unsteady flow effects has received less attention. Typically, the thrust and torque coefficients could be written in a more general manner as $C_{T}=k \cdot f(\alpha, \beta, \omega, \dot{\alpha}, \dot{\beta}, \dot{\omega})$, with $\alpha, \beta$, and $\omega$ as the three possible solid rotation angles and $\dot{\alpha}, \dot{\beta}$, and $\dot{\omega}$ as their corresponding angular velocities.

A rotor blade can experience different types of oscillating motions as a response to unsteady aerodynamic forces and fluid-structure interactions (FSIs). As a first approach, these motions can be described as three solid rotations about the axis oriented radially, azimuthally, and perpendicularly to the mean blade path, which are referred to as pitching, flapping, and surging motions. For lowamplitude oscillations, in the linear, attached flow regime, the resulting unsteady aerodynamic forces and blade motion can be predicted using conventional, potential flow theory [1,2]. However, when the effective angle of attack of the blade exceeds the static stall angle of the airfoil blade section, leading-edge flow separation occurs. Such nonlinear phenomena require high-fidelity numerical simulations or measurements to predict the complex physics that lead to drastic changes in the aerodynamic performance of the blade. In these specific cases, the blade motion is correlated with the time scale of large-scale coherent vortices being formed at the leading edge of the blade.

Although an uncontrolled blade motion most presumably results in a decrease in aerodynamic performance, a controlled (forced) motion could potentially have a beneficial impact. This was first suggested by van Holten [3] , who introduced the concept of a flapping rotor on a medium-scale rotorcraft model: the flapping motion was powered, whereas the rotating motion was induced by the flapping motion. Such a mechanism has the potential to annihilate the rotating reaction torque, eliminating the need for a tail rotor. This concept was further investigated on a microscale rotor [4,5], sometimes with the ability to couple both flapping and (active or passive) pitching motions, and with powered or induced rotation. It recently gained considerable interest with a significant amount of work [6-12]. Overall, these studies suggested that thrust could significantly be enhanced with respect to conventional rotors yet with lower efficiency. Similar conclusions were raised for a pitching rotor [13], where the rotating blade undergoes a pitching motion about a spanwise axis (without flapping motion). In a general manner, the research on improved kinematics is a potential way to increase the performance of propulsive systems [14].

The numerical simulation of such FSIs requires us to tackle the coupling between a computational fluid dynamics solver and a computation structural dynamics solver, including the difficult task 
of remeshing or grid morphing [15]. An alternative approach is to use a lattice-Boltzmann method $(\overline{\mathrm{B} M})$ coupled with a finite element method for the structural solver $[\underline{16}, \underline{17}]$ or an immersed boundary method [18].

The objective of the present work is thus to study the influence of unsteady flow effects on the performance of a rotor, which is adapted to the propulsion of micro air vehicles (MAVs). Several challenges are associated to this objective: the numerical prediction of such unsteady flows (leading-edge vortex, massive separation, and turbulence) remains difficult, and the unsteady displacement of the blade (due to forced motion or dynamic response to unsteady aerodynamic forces) requires adapted numerical methods. To address these difficulties, the present work relies on the development of FSI capabilities in a LBM code to take advantage of the immersed boundary approach. The first part of this paper presents the implementation of such a FSI by coupling the equation of the dynamics with the aerodynamic flow solver. The method is validated on a vortex-induced vibration test case at a very low Reynolds number, for which reference data are available. Then, these methods are used to study the influence of forced motions as flapping, pitching, and surging on the rotor performance. A model is then developed to predict the influence of these kinematics on the rotor thrust. Finally, the analysis is extended to cases where blade oscillating motions are induced by FSIs.

\section{Test Case and Numerical Methods}

\section{A. Geometry and Operating Conditions}

The test case is a two-bladed rotor of diameter $D=0.250 \mathrm{~m}$ operating in hover and designed to be representative of a typical MAV rotor. The main characteristics of the rotor are reported in Table 1 . The rotor is composed of two untwisted flat plates. The span $R$, the chord $C$, and the thickness $h$ of the blade are $0.100,0.025$, and $0.001 \mathrm{~m}$, respectively. The distance between the hinges of the two blades is set to two chords. The angle of attack of the profiles is initially set to $\alpha_{0}=15 \mathrm{deg}$, as shown in Fig. 1 . The rotation speed $\Omega$ of the rotor is set to $3960 \mathrm{rpm}$, corresponding to a Mach number at tip of 0.151 . The data presented in this paper are normalized using a standard atmosphere, with a temperature of $T_{0}=293 \mathrm{~K}$ and a static pressure of $p_{0}=101,325 \mathrm{~Pa}$.

\section{B. Structural Properties of the Blade}

Regarding the dynamic response of the blade to aerodynamic forces, the main rotations of interest are the pitch $\alpha$ and flap $\beta$. As a first approximation, the blade is considered as an articulated rigid plate. To predict the dynamic behavior of the blade, it is thus necessary to estimate the values of the rotational stiffness and the moment of inertia related to the blade. The dimensions of the blade are $R, C$, and $h$, as reported in Table 1 . The blade is made of epoxy $\left(\rho_{S}=1.5 \times 10^{3} \mathrm{~kg} \cdot \mathrm{m}^{-3}\right)$ and is assumed to be homogeneous. The calculations of the moment of inertia for the pitch and flap are done with solid cuboid formulas, as detailed in the Appendix. The stiffnesses in pitching $K_{\alpha}$ and flapping $K_{\beta}$ are then estimated by considering the shear modulus of the epoxy $\left(G_{\text {epoxy }}=1.25 \mathrm{GPa}\right)$ and are shown in Table $\underline{2}$.

Table 1 Characteristics of the rotor test case

\begin{tabular}{lc}
\hline \hline Parameter & Value \\
\hline Number of blades & 2 \\
Rotation rate $\Omega$ & $414.69 \mathrm{rad}^{-1}$ \\
Rotor diameter $D$ & $0.250 \mathrm{~m}$ \\
Radius at tip $R$ & $0.125 \mathrm{~m}$ \\
Radius at root $r$ & $0.025 \mathrm{~m}$ \\
Blade chord $C$ & $0.025 \mathrm{~m}$ \\
Blade span $R_{S}$ & $0.100 \mathrm{~m}$ \\
Blade thickness $h$ & $0.001 \mathrm{~m}$ \\
Reynolds number $R e$ & $0.86 \times 10^{5}$ \\
\hline \hline
\end{tabular}

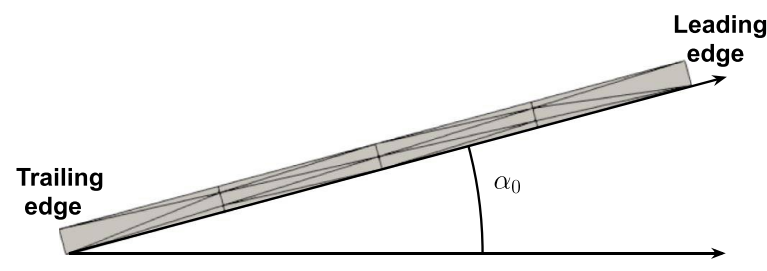

Fig. 1 Lateral view from the hub of the blade.

\section{Unsteady RANS}

The three-dimensional unsteady Reynolds-averaged NavierStokes (URANS) equations are solved under their incompressible form using the StarCCM+ commercial code. An overset grid approach is used that allows each blade mesh to move, following prescribed rotating and sinusoidal pitching motions within a stationary background mesh. The structured mesh consists of 4.7 million hexahedral cells ( 0.9 million for each blade mesh and 2.9 million for the background mesh) enclosed within a box domain of width $20 R$ and height $50 R$ (see Fig. 2). The boundary conditions upstream and downstream of the rotor are implemented as pressure Dirichlet conditions, whereas the periphery of the domain is defined using a slip-wall condition. The blades are modeled as nonslip surfaces. The blade mesh is moved with a time step corresponding to a Courant-Friedrichs-Lewy number below 10. Therefore, the time step is adjusted to pitching motion parameters with at least 720 time steps per rotating period. Both spatial and temporal discretizations are achieved using second-order schemes. Momentum and continuity equations are solved in an uncoupled manner using a predictorcorrector approach. Finally, the Spalart-Allmaras model is employed for turbulence closure with maximum $y^{+}$values on the order of six (with an average value close to two).

\section{LES-LBM}

The large-eddy simulation (LES) is performed by means of a lattice-Boltzmann method, which already demonstrated its capability to solve flows for low-Reynolds-number rotors [19]. Beyond its computational performance, the main advantage of the LBM is that the method is stable without artificial dissipation, which makes the method equivalent to solving the Navier-Stokes equations with a high-order numerical scheme. Its drawback is that it requires the use of Cartesian grids. To counterbalance this limitation, the walls are represented through an immersed boundary approach [20]. The main advantage of this method is that the position of the wall can be easily updated at each time step, which makes this technique well suited to unsteady blade motion. The LBM considers the discrete Boltzmann equation, which is a statistical equation for the kinetics of gas molecules, instead of directly solving the Navier-Stokes equations. As detailed in Refs. [21,22], the governing equations consider the probability $f_{i}(x, t)$ to have a set of particles at location $x$ and time $t$, with velocity $c_{i}$ :

$$
f_{i}\left(x+c_{i} \delta t, t+\delta t\right)=f_{i}(x, t)+\Omega_{i j}(x, t)
$$

for $[0<i, j<N]$, where $c_{i}$ is a discrete velocity of a set of $N$ velocities, and $\Omega_{i j}$ is an operator representing the internal collisions of pairs of particles. In this work, the kinetic scheme is based on a D3Q27 formulation that ensures the conservation of mass and momentum. The collision operator is represented by a single relaxation time model, and a regularization technique is applied to increase the stability and accuracy of the method $[23,24]$. The

Table 2 Structural properties of a blade

\begin{tabular}{lcc}
\hline \hline & Pitch & Flap \\
\hline Moment of inertia $I, \mathrm{~kg} \cdot \mathrm{m}^{2}$ & $2.9 \times 10^{-7}$ & $2.8 \times 10^{-5}$ \\
Polar moment of area $J, \mathrm{~m}^{4}$ & $2.3 \times 10^{-9}$ & $6.5 \times 10^{-7}$ \\
Stiffness $K, \mathrm{~N} \cdot \mathrm{m}$ & 30 & 8100 \\
\hline \hline
\end{tabular}




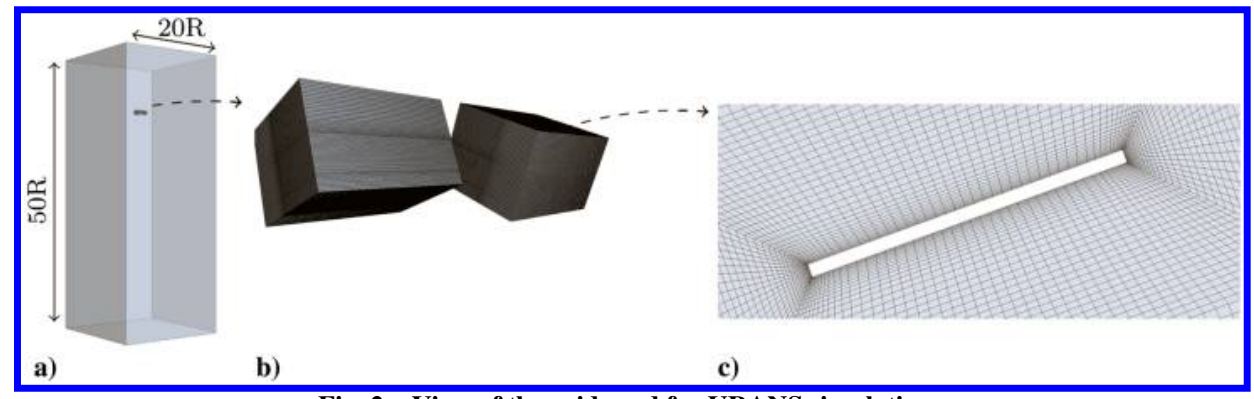

Fig. 2 View of the grid used for URANS simulations.

regularization step ensures a LES formulation without a subgrid scale model [25].

Previous works [26] have shown that the convergence of thrust and torque requires us to achieve a grid resolution corresponding to $\Delta x / C=0.01-0.015$. The dimension of the first cell in the direction normal to the wall is thus set to $\Delta x / C=0.015$, corresponding to $\overline{y^{+}} \approx 50$. Far from the wall, the cell size is increased by means of a hierarchical grid refinement approach with five grid levels (from one grid to the next grid, both the time step and the spatial step are increased by a factor of two). The total number of grid points for the full mesh is $143.5 \times 10^{6}$ (with $50 \%$ of the points located in the vicinity of the rotor disk in the first grid level). A full rotation of the rotor is discretized with 20,100 time steps. The typical computational time needed to achieve one rotation of the rotor is $1500 \mathrm{hCPU}$ (with 120 cores of a scalar supercomputer). About 10 rotations are simulated to achieve a stabilized operating point.

\section{Modeling of Fluid-Structure Interactions}

\section{A. Development of FSI Capabilities}

A monolithic aeroelastic flow solver is developed to maintain the computational performance of the LBM code. The approach relies on the use of generalized coordinates to represent each blade of the rotor as an articulated plate. As a first step, a simple dynamical model is considered, based on a classical second-order dynamic model for the structure deformation, as follows:

$$
I_{q} \frac{\mathrm{d}^{2} q(t)}{\mathrm{d} t^{2}}+D_{q} \frac{\mathrm{d} q(t)}{\mathrm{d} t}+K_{q} q(t)=M_{q}(t)
$$

with $q$ as a generalized coordinate of the system, $I_{q}$ as the moment of inertia with respect to the axis of rotation of the coordinate $q, D_{q}$ as a damping factor, $K_{q}$ as the stiffness of the structure, and $M_{q}$ as the sum of external moments applied to the system with respect to the axis of rotation of coordinate $q$. Three generalized coordinates are used to represent the displacement of the blade:

1) The pitching angle $\alpha$ around the spanwise axis is located a quarter-chord away from the leading edge (corresponding to the aerodynamic center). The corresponding angular velocity is noted as $\dot{\alpha}$ in Fig. 3 a.
2) The flapping angle $\beta$ is around the blade hinge, horizontal, perpendicular to the spanwise axis, and with its origin at the rotor hub. The flapping angular velocity is noted as $\dot{\beta}$ in Fig. $3 \mathrm{~b}$.

3 ) The surging angle $\omega$ is around the main rotor axis. The angular velocity is noted as $\dot{\omega}$ in Fig. 3c (this movement corresponds to a variation of the rotation speed $\Omega$ ).

The integration of Eq. (2) recovers the previous quantities that, combined with the main rotational velocity $\Omega$, returns the absolute angular velocity of each discrete surface point.

\section{B. Numerical Implementation}

To impose the unsteady displacement of the blade, the following algorithm is implemented in the flow solver:

1) After the calculation of equilibrium distributions and before the collide and stream steps, every Lagrangian surface particle is assigned with a velocity function and all forces on the particles are reset to zero.

2) Particles are advanced everywhere to their new position.

3) The immersed boundary algorithm is applied until the compatibility criterion is met (following an iterative process that requires typically four to six iterations).

\section{Determination of the Velocity Functions}

Typically, two types of velocity function can be imposed: 1) a forced motion [the kinematic of the blade is know a priori, and so it is not necessary to solve Eq. (2)], and 2) a dynamic response that requires us to solve Eq. (2) to know the new displacement velocity of the blade.

\section{Forced Motion}

The forced motion model imposes a periodic motion around a secondary axis (e.g., hinge, spanwise axis or, in the case of surging, the same rotor axis) that is superimposed to the main rotation of the blade. This approach is similar to that presented in Ref. [27]. A sinusoidal angular velocity $\dot{q}$ is chosen for the corresponding generalized coordinate:

$$
\dot{q}=-\omega_{m} q_{\max } \cos \left(\omega_{m} t\right)
$$

where $\omega_{m}$ is the motion pulsation, and $q_{\max }$ is the amplitude of the motion. The velocity function given to Lagrangian points

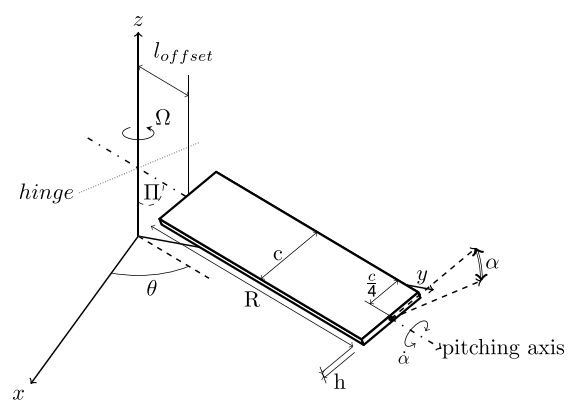

a) Pitching

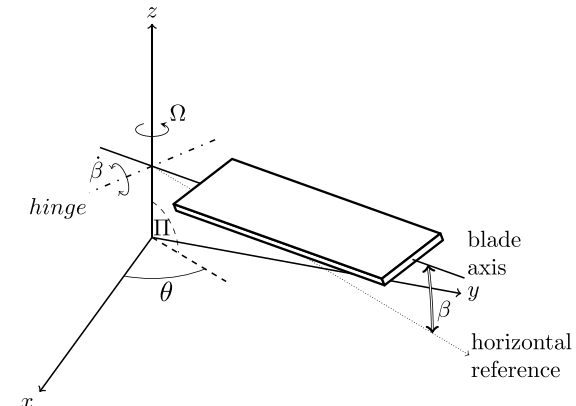

b) Flapping

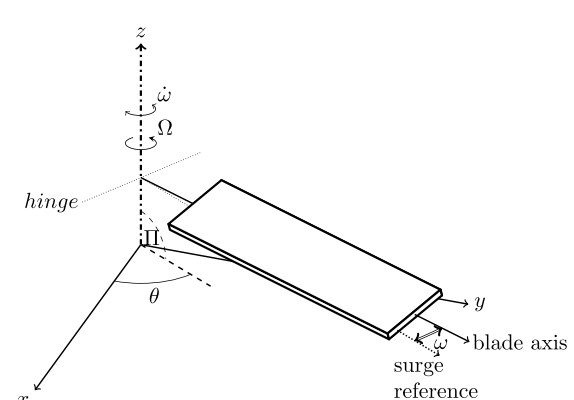

c) Surging

Fig. 3 View of the generalized coordinate system used to describe blade movement: a) pitching model with offset $l_{\text {offset }}$ with respect to main rotation axis, b) flapping model (blade rotates around hinge), and c) surging model (blade rotates with nonconstant rotational speed). 
corresponds to the sum of the velocities due to the two successive rotations, with angular velocities $\Omega$ and $\dot{q}$ in a single time step.

\section{Dynamic Response}

The model solving the dynamic interaction between the fluid and the structure requires us to integrate Eq. (2), which is done using a fourth-step Runge-Kutta scheme. This integration is performed at the coarsest level of the numerical simulation, whereas the immersed boundary algorithm is updated at the finest level. This results in a constant angular acceleration at the chosen generalized coordinate during the whole "coarse" time step duration, and the angular velocity of the chosen generalized coordinate evolves linearly. Introducing the state variable $\boldsymbol{Q}=[q \dot{q}]$ and rearranging Eq. (2), a system of the first order is retrieved:

$$
\frac{\mathrm{d}}{\mathrm{d} t} \boldsymbol{Q}=\left[\begin{array}{c}
\dot{q} \\
\frac{M}{I}-\frac{K}{I} q-\frac{D}{I} \dot{q}
\end{array}\right]=A
$$

The inertia, damping, stiffness, and force terms are, respectively (in the case of rotation), the mass moment of inertia, the rotational damping, the rotational stiffness, and the torque around the axis of rotation.

\section{Verification and Validation}

The FSI capabilities implemented in the LES-LBM flow solver are first validated to verify the accuracy of the method. Here, a two-step verification and validation procedure is thus performed: first, FSI capabilities are validated for a simple test case (low-Reynoldsnumber cylinder flow); then, the aerodynamic capabilities of the LES-LBM flow solver are validated for a rigid rotor configuration.

\section{A. Application to a Vortex-Induced Vibration Case}

The test case used is based on the configuration investigated by Singh and Mittal [28], which considers a cylinder in a vortex-induced vibration (VIV) regime. The geometry is a cylinder of diameter $D=1 \mathrm{~m}$ at a Reynolds number of $R e=100$. The normalized mass of the cylinder of $m^{*}=4 m /\left(\pi \rho_{f} D^{2}\right)$ is set to $m^{*}=10$. The value of the cylinder stiffness $k$ is adjusted in order to match the desired value of the normalized velocity $U^{*}$ defined as

$$
U^{*}=\frac{V_{\infty}}{(1 / 2 \pi) \sqrt{(k / m)} D}
$$

with $V_{\infty}$ as the infinite flow velocity. The value of $U^{*}$ represents the ratio between the characteristic flow frequency $\left(V_{\infty} / D\right)$ and the cylinder natural frequency $(=1 /(2 \pi) \sqrt{k / m})$. For this application, due to the low value of the Reynolds number, the subgrid scale model is deactivated so that the flow solver is run in full direct numerical simulation mode. Velocity is imposed at the inlet, and static pressure is imposed at the outlet. Slip conditions are set on the upper and lower boundaries. As in Ref. [28], the structural damping is neglected $[D=0$ in Eq. (2) $]$.

Particular attention is brought to the convergence of aerodynamic coefficients with respect to the discretization and the domain size. Numerical simulations have been performed with 60, 80, and 120 grid points per cylinder diameter. Based on these results, the grid spacing and the time step requirements to achieve the convergence of aerodynamic coefficients are $\Delta x=0.0125 \mathrm{~m}$ and $\Delta t=6.25 \times$ $10^{-5} \mathrm{~s}$, respectively [corresponding to $D / \Delta x=80$ and $D /(\Delta t \times$ $\left.\left.V_{\infty}\right)=1600\right]$. Several sizes of the domain are also tested, such as $L_{x} \times L_{y}=20 D \times 20 D, 30 D \times 30 D$, and $40 D \times 40 D$. The minimum size of the domain to ensure the convergence of aerodynamic coefficients corresponds to $L_{x}=40 \mathrm{D}$ and $L_{y}=40 \mathrm{D}$ (with the cylinder located at the center of the domain). The flow physics related to this test case is 2-D; however, only the three-dimensional (3-D) formulation of the immersed boundary available in the code is compatible with an unsteady displacement of the walls, and so a 3-D grid is necessary. With such requirements, the 3 -D grid is made of $17.3 \times 10^{6}$ points.

For the fully rigid case $\left(U^{*} \rightarrow 0\right)$, a vortex shedding develops behind the cylinder. The values predicted with the LBM for the drag coefficient $C_{D}$, the rms lift coefficient $C_{L_{\mathrm{RMS}}}$, and the Strouhal number $S t$ are $1.39,0.24$, and 0.168 , respectively. These values are compared with those obtained in the literature, e.g., $C_{D}=1.31$, $C_{L_{\mathrm{RMS}}}=0.25$, and $S t=0.163$ [28] or $C_{D}=1.35, C_{L_{\mathrm{RMS}}}=0.33$, and $S t=0.167$ [29]. The values predicted with the LBM are thus in good agreement with the results already reported for the same configuration. When increasing the value of $U^{*}$, the cylinder starts to oscillate due to the unsteady lift generated by the vortex shedding. The evolution of the cylinder displacement with respect to the normalized time $t^{+}=t \cdot V_{\infty} / D$ and the instantaneous flowfield colored with vorticity for two values of $U^{*}$ are shown in Fig. 4 . The time $t^{+}=0$ corresponds to the instant where the cylinder is let free to oscillate in the flow. When the natural frequency of the cylinder is close to the vortex shedding frequency, the unsteadiness of the lift coefficient suddenly rises due to the lock-in phenomenon.

The maximum displacement of the cylinder $y / D$ with respect to the parameter $U^{*}$ is shown in Fig. 5. These data are compared to those obtained by Singh and Mittal [28] . The shape of the curve $y / D=$ $f\left(U^{*}\right)$ is correctly reproduced by the present numerical simulations, with a good estimation of the maximum displacement ratio $y / D$ (the discrepancy on the maximum displacement is $3 \%$ as compared to the reference data of Singh and Mittal). The present numerical method predicts that the lock-in phenomenon starts for $U^{*}>4.5$. From

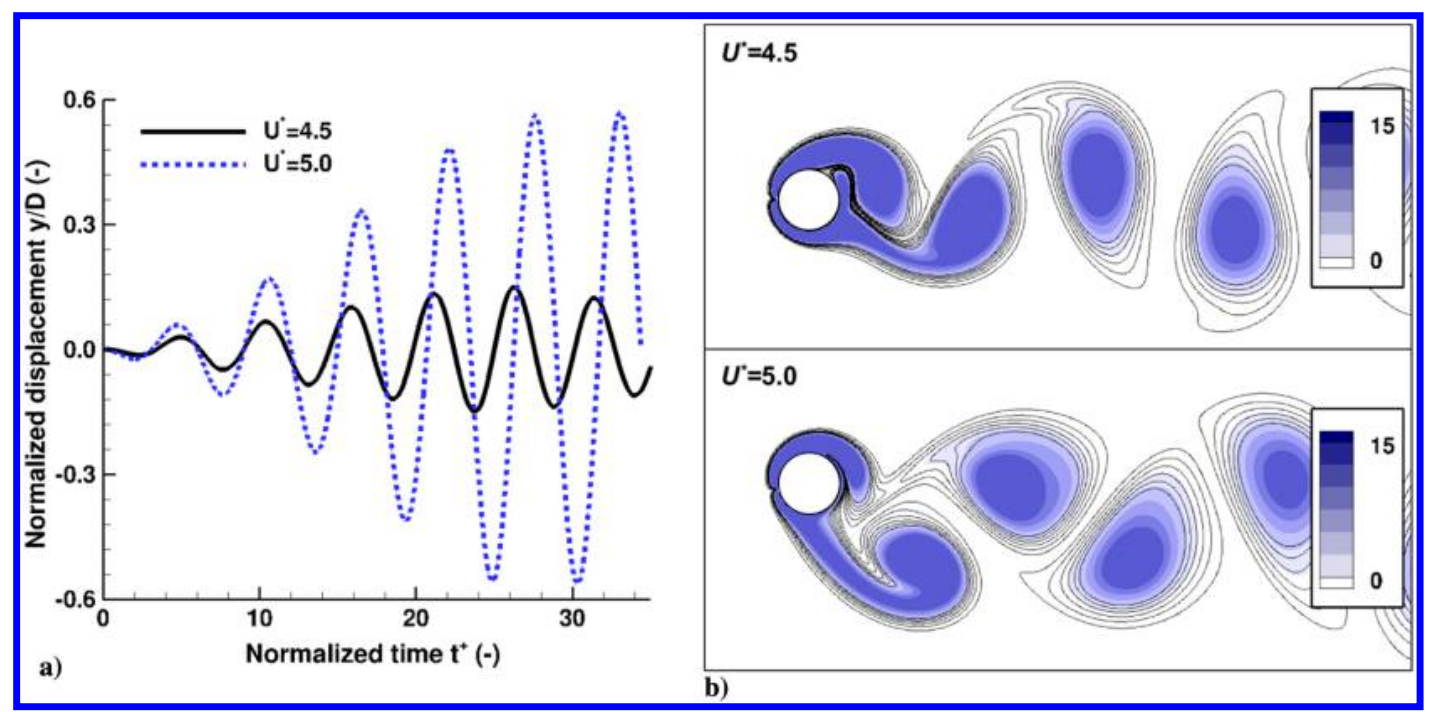

Fig. 4 Simulations with present FSI-LBM flow solver for two different normalized velocities $U^{*}:$ a) normalized displacement $y / D$, and b) instantaneous flowfield colored with vorticity. 


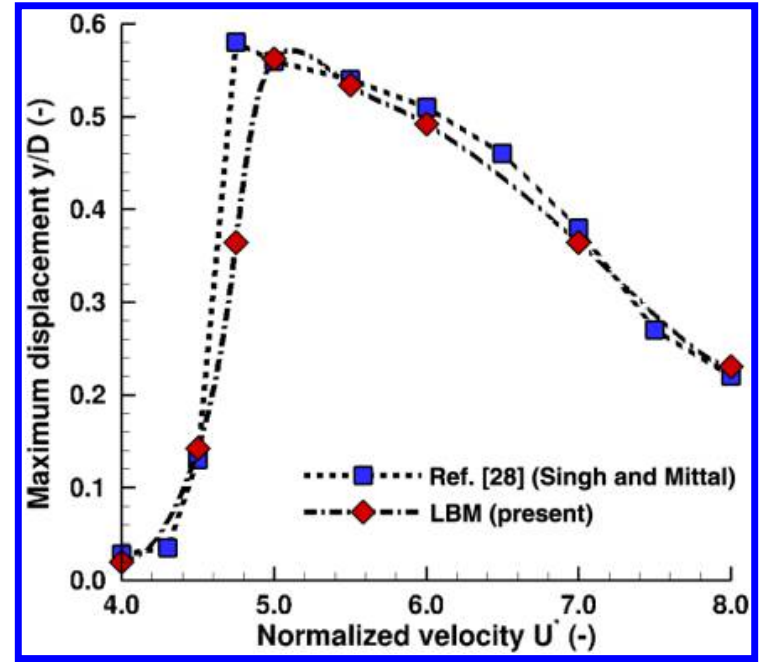

Fig. 5 Prediction of maximum displacement $y / D$ of cylinder at $R e=$ 100 with respect to normalized velocity $U^{*}$ and comparison with the results of Singh and Mittal [28].

$U^{*}=4.5$ to $U^{*}=5.0$, the maximum displacement of the cylinder increases rapidly, with a small shift of about $2 \%$ as compared to the reference data (e.g., for $U^{*}=4.75$, the value of $y / D=0.36$ corresponds to the one observed at $U^{*}=4.65$ in the reference data). When the value of $U^{*}$ is still increased, the maximum displacement of the cylinder progressively decreases similarly to the reference.

\section{B. Prediction of the Rotor Performance}

The second step is to validate the capability of LES-LBM to accurately estimate 1) the global performance of the rotor as compared to experimental data, and 2) the effect of a forced motion as compared to URANS predictions. The torque and thrust coefficients, $C_{Q}$ and $C_{T}$, are defined as

$$
C_{T}=\frac{\bar{T}}{(1 / 2 \times 16) \rho(\Omega \cdot D)^{2} \pi D^{2}}
$$

and

$$
C_{Q}=\frac{\overline{Q \cdot \Omega}+\overline{Q_{i} \cdot \dot{\omega}_{i}}}{(1 / 2 \times 32) \rho \Omega^{3} \pi D^{5}}
$$

To allow a fair comparison between all the configurations in the case of forced motion, the torque coefficient takes into account for two contributions: 1) the power needed to impose the rotation $P_{\Omega}=\overline{Q \cdot \Omega}$; and 2) the power to impose the secondary motion $P_{\dot{\omega}}=\overline{Q_{i} \cdot \dot{\omega}_{i}}$ (with $Q_{i}$ and $\dot{\omega}_{i}$ as the instantaneous torque and displacement velocity related to the forced motion). All comparisons are done at the same rotation speed of $\Omega=414.69 \mathrm{rad} / \mathrm{s}$.

The evolution of $C_{Q}$ with respect to $C_{T}$ is shown in Fig. 6 for two configurations: 1) the reference configuration (pure rotation), and 2) a case where a forced motion of pitching is superimposed to the rotational speed $\left(\omega_{m}=3 \Omega\right.$ and $q_{m}=0.1724$, corresponding to a variation of $\pm 9.9 \mathrm{deg}$ around the average pitch angle). Experimental data for the reference configuration (pure rotation) have been obtained in the frame of a previous campaign [30]. Three different blade angles are considered for the LES-LBM: $\theta=10 \mathrm{deg}$, $\theta=15 \mathrm{deg}$, and $\theta=20 \mathrm{deg}$. The comparison with other data (measurements and URANS) shows that the LES-LBM is able to predict the evolution of the torque coefficient with respect to the thrust coefficient (Fig. 6a). For $\theta=15 \mathrm{deg}$ (pure rotation case), the discrepancies on thrust between the URANS, the LES-LBM, and the measurements is 7 and $14 \%$, respectively. Both the URANS and LES-LBM correctly estimate the $C_{Q} / C_{T}$ ratio. The figures of merit are 0.611 (measurements), 0.647 (URANS), and 0.616 (LES-LBM). This comparison validates the capability of the present LES-LBM to predict the rotor performance with a good accuracy; the figure of merit is especially estimated with an error lower than $1 \%$.

For the case with a forced pitching motion, the URANS predicts increases of the thrust and torque by 1 and 24\%, respectively. For the same configuration, the LES-LBM predicts increases of the thrust and torque by 4 and $21 \%$, respectively, which are in good agreement with the URANS results. This comparison is satisfying because the flow is affected with alternative separation and reattachment phases, which are known to be challenging to predict for numerical simulations. The conclusion of this section is that the LES-LBM is able to predict the effect of complex motion, like pitching, on the rotor performance.

\section{Forced Motion: Pitching, Flapping, and Surging}

A. Influence on the Rotor Performance

The three solid rotations (pitching, flapping, and surging) have very different effects on the flow that is seen by the blade. A scheme is shown in Fig. 7 to explain how each forced motion will modify the flow condition seen by the blade. As indicated by Eq. (3), if only harmonic motions are considered, each elementary motion depends on only two parameters: the amplitude $q_{\max }$, and the frequency $\omega_{m}$. The forced motion frequency can then be compared to a characteristic frequency of the flow (e.g., based on the time needed to travel from the leading edge to the trailing edge of the blade) to define a reduced velocity $U^{*}$ as

$$
U^{*}=\frac{\Omega \cdot \bar{r}}{\omega_{m} C}
$$

with $\bar{r}$ as the radius at midspan and $C$ as the chord. To ensure interactions between the forced motion and the flow, the value of $U^{*}$
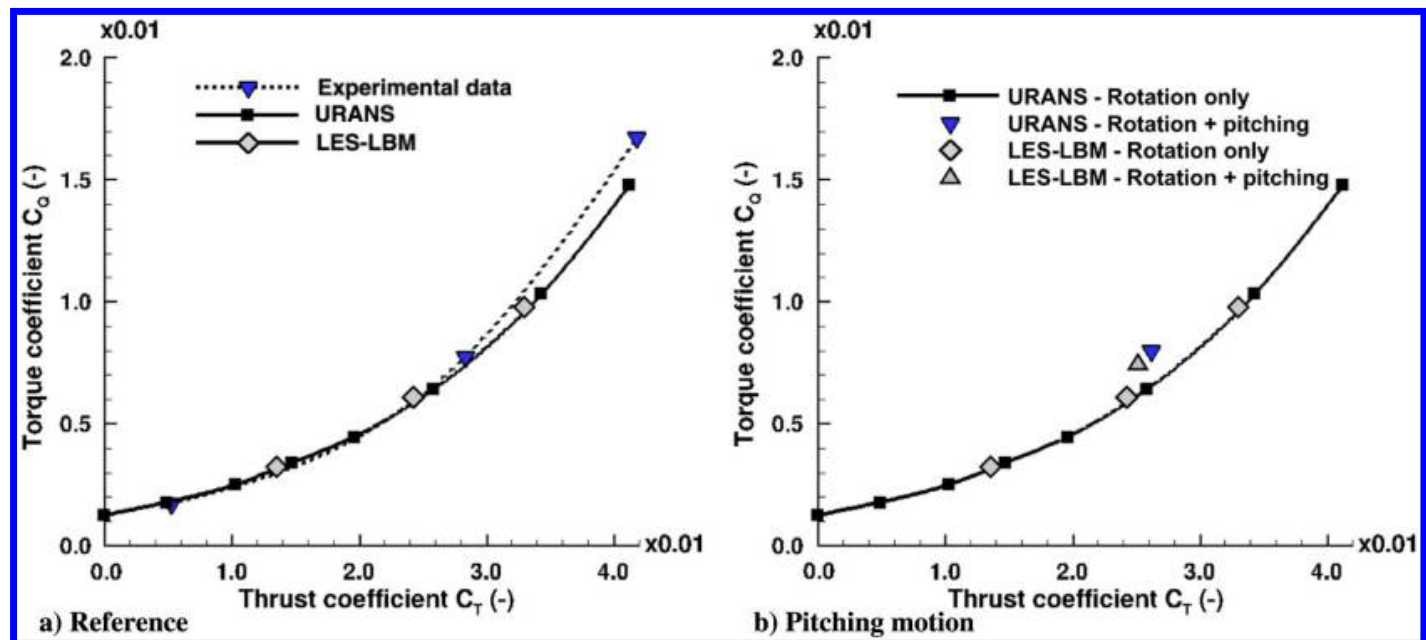

Fig. 6 Prediction of torque coefficient $C_{Q}$ with respect to thrust coefficient $C_{T}$ : a) comparison with measurements (from Ref. [30]), and b) influence of pitching on aerodynamic performance. 
should be of the magnitude order of one: for $U^{*} \ll 1$, the flow does not have the time to adapt (the forced motion effects will be filtered by the flow), whereas for $U^{*} \gg 1$, the flow will adapt very rapidly as compared to the forced motion velocity, corresponding to a succession of quasi-steady states.

The simplest forced motion is the pitching motion (Fig. 7a): the blade rotates around its center, located at the quarter-chord, and so the flow conditions at the inlet alternatively vary between $\left(\alpha_{0}-\Delta \alpha\right)$ and $\left(\alpha_{0}+\Delta \alpha\right)$. The effect of the parameter $q_{\max }$ is to directly set the minimum and maximum angles that will be seen by the blade. The influence of the frequency $\omega_{m}$ is more subtle: by inducing an angular speed at the leading edge, it modifies the effective angle of attack seen by the profile. This effect is added to the geometric blade angle.

The flapping is a complex motion composed of two parts (Fig. 7b): first, a downstroke movement, where the blade moves in the same direction than the induced velocity; then, an upstroke movement, where the blade moves in the direction opposite to the induced velocity. Because the flapping motion corresponds to a rotation around the hinge, a part of the flapping velocity component is added (downstroke) or deducted (upstroke) from the main rotational speed. The result is that, contrary to the pitching case, this movement is not exactly symmetric regarding the variation of the angle of attack. If the velocity of the blade displacement is higher than the induced velocity, this would result in an inversion of the suction and pressure sides.

The surging motion corresponds to a variation of the rotational speed (Fig. 7c). Alternatively, the blade decelerates, and then it accelerates. As shown on the scheme, assuming a constant induced velocity that is lower than the rotational speed, the effect of the deceleration on the angle of attack is more important than the effect of the acceleration. During the deceleration and acceleration phases, the angle of attack is decreased and increased, respectively.

An efficient comparison of the three motions is not straightforward because it requires us to know a priori the value of the induced velocity (that depends on the global performance of the rotor). Assuming that one of the most important parameters is the variation of the angle of attack, an effort has been made to ensure that this parameter remains of the same magnitude order when comparing all three motions.

The parameters used for each of the three forced motions are indicated in Table 3 . A frequency corresponding to a reduced velocity of 1.0 has been chosen for all forced motions to ensure a contribution of unsteady flows to the rotor performance.

The effect on the torque and thrust coefficients is shown in Fig. 8. The three different motions lead to an increase of the thrust for the same rotation speed $\Omega$ : by $+4 \%,+14 \%$, and $+45 \%$ for the pitching,

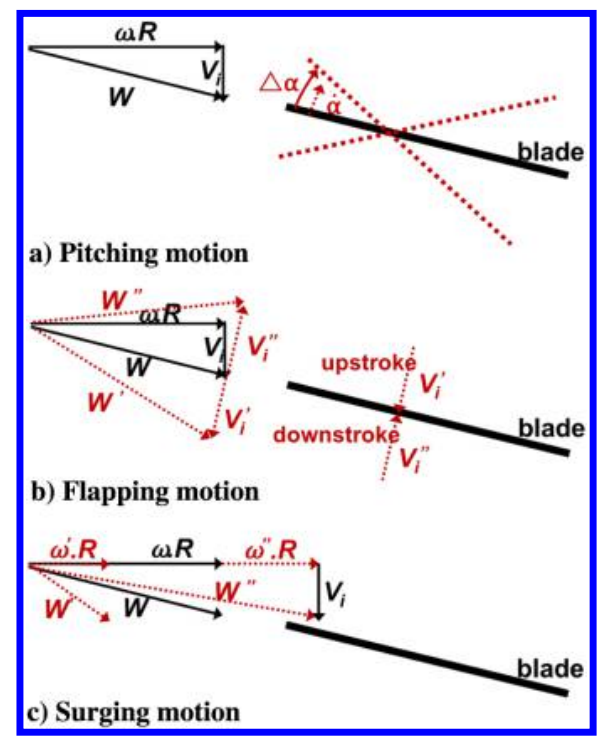

Fig. 7 Explanation of influence of three different forced motions on flow conditions seen by blade: a) pitching, b) flapping, and c) surging. $V_{i}$ is induced velocity, and $W$ is velocity in relative frame (resulting from combination of rotation speed and induced velocity).
Table 3 Parameters of the forced motions

\begin{tabular}{lccc}
\hline \hline & Pitch & Flap & Surge \\
\hline Amplitude $q_{\mathrm{max}}, \mathrm{rad}$ & 0.1724 & 0.0912 & 0.2314 \\
Frequency $\omega_{m}, \mathrm{rad} / \mathrm{s}$ & $3 \Omega$ & $3 \Omega$ & $3 \Omega$ \\
Reduced velocity $U^{*}$ & 1.0 & 1.0 & 1.0 \\
\hline \hline
\end{tabular}

flapping, and surging motion, respectively. However, a penalty on the torque is observed for all three motions as compared to the pure rotation case. The data reported in Table $\underline{4}$ show that, except in the case of pitching, the power needed to actuate the blade and impose the forced motion is found to be significant (around $40 \%$ in the case of flapping and surging). This means that some improvements could be done to optimize the kinematics of the blade and reduce this penalty. In the case of surging, the penalty is moderate as compared to the increase in thrust (the new operating point is close to an operating point corresponding to $\alpha=20 \mathrm{deg}$ ).

The periodic increase of thrust is correlated with the periodic motion of the rotor, as shown in Fig. 9. By convention, the rotation speed $\Omega$ is negative, as well as the mean pitching angle, and so a negative component is added to the mean angle and a positive component is retrieved to the mean angle (e.g., the downstroke step corresponds to an increase of the flapping angle $\beta$ ). For the reference case, the thrust coefficient varies by \pm 0.001 around its averaged value. In the case of forced motions, the thrust coefficient varies by $\pm 0.034, \pm 0.021$, and \pm 0.038 in the case of pitching, flapping, and surging, respectively. The time lags between the variation of the airfoil position and the variation of the thrust are about $0.03 T, 0.08 T$, and $0.04 T$ in the cases of pitching, flapping, and surging, respectively (with $T$ as the period of revolution of the rotor). In the case of surging, the extrema of the surging motion in Fig. 9c correspond to an instantaneous rotation speed $\Omega_{S}$ that is equal to the mean rotor rotation speed $\Omega$. These results can be summarized as follows:

1) For pitching, the thrust is correlated with the blade position, and the maximum thrust is achieved a bit before the blade reaches its maximum pitch angle (at maximum pitch angle, the thrust starts to decrease due to a massive flow separation).

2) For flapping, the thrust is correlated with the blade velocity, and the maximum thrust is achieved during the downstroke step when the blade reaches its maximum displacement speed; during half of the upstroke step, the thrust becomes negative.

3) For surging, the thrust is correlated with the blade velocity, the maximum thrust is achieved after the blade reaches its maximum rotation speed (about $1.7 \Omega$ ), and the minimum thrust is achieved after the blade reaches its minimum rotation speed (about $0.3 \Omega$ ).

Instantaneous flowfields related to the reference case (pure rotation) are shown in Fig. 10. The four instants describe one-third of the rotor revolution. An intense leading-edge vortex develops along

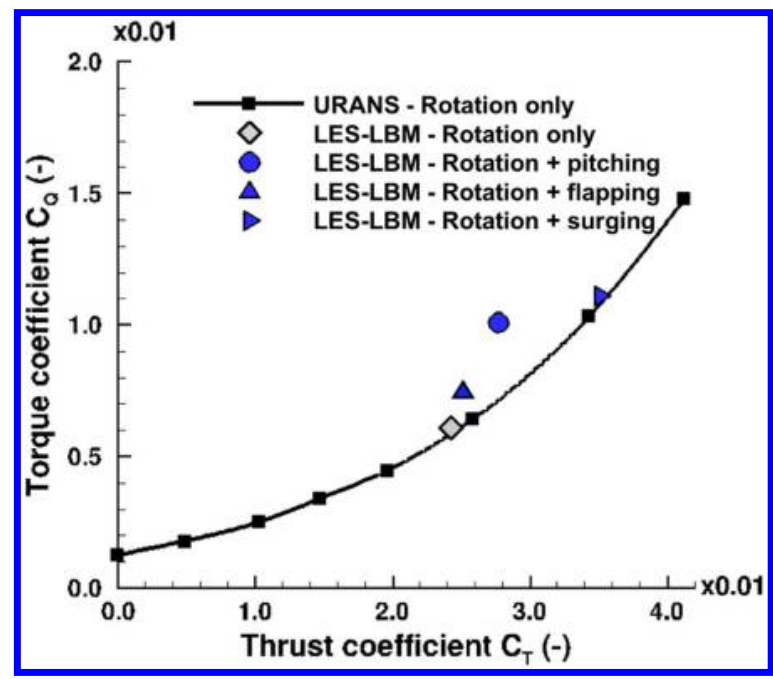

Fig. 8 Comparison of the torque coefficient $C_{Q}$ with respect to thrust coefficient $C_{T}$ obtained by imposing different forced motions. 
Table 4 Contributions to the torque coefficient

\begin{tabular}{lccc}
\hline \hline & Pitch & Flap & Surge \\
\hline Total torque coefficient & 0.0074 & 0.0101 & 0.0111 \\
Due to rotation, $\%$ & 99 & 61 & 55 \\
Due to the forced motion, $\%$ & 1 & 39 & 45 \\
\hline \hline
\end{tabular}

the blade span, leading to a massive separation close to the blade tip. Such a flow phenomenon has already been reported in the literature for similar low-Reynolds-number rotors [19,31,32]. The picture shown in Fig. 10b confirms that the flow is separated at $r / R=0.8$, generating intense vortices behind the trailing edge. These vortices are responsible for a high level of turbulent activity that impacts the leading edge of the following blade. Moreover, the leading-edge vortex exhibits an unsteady behavior with periodic ejection of vortices along the blade.

\section{B. Effect of Pitching}

Instantaneous flowfields for the pitching case are plotted in Fig. 11 at four different instants, describing a period of time associated to the pitching motion (corresponding to one-third of the rotor revolution). The arrow at the blade leading edge indicates the direction in which the blade is moving, the dashed-dotted line indicates the two extremum positions, and the dotted line shows the mean position. The chosen instants are shown in Fig. 9 and are identical for all kinematics. The flow at $t=t_{0}$ corresponds to a position close to the highest value of pitching angle $\left(\alpha=\alpha_{0}+\Delta \alpha \approx 25 \mathrm{deg}\right)$. The boundary layer on the suction side is fully separated, which explains the reduction of thrust at this position. When the incidence is progressively reduced, the flow reattaches (instant $t=t_{2}$ ). However, the influence of the leading-edge vortex is still visible, even at the lowest incidence. When the blade returns close to its original position $\left(t=t_{3}\right)$, the intensity of the leading-edge vortex re-increases. This vortex is then ejected toward the trailing edge when the incidence is further increased. The vorticity associated to the leading-edge vortex is responsible for the weak increase of the thrust coefficient as compared to the pure rotation case.

\section{Effect of Flapping}

Instantaneous flowfields for the flapping case are plotted in Fig. 12 at four different instants, describing a period of time associated to the flapping motion. The arrows indicate the directions in which the blade is moving, the dashed-dotted lines indicate the two extremum positions, and the dotted lines show the mean positions. The flow at $t=t_{0}$ corresponds to the beginning of the downstroke step. At this position, the vorticity associated to the leading-edge vortex is of the same magnitude order as compared to the pure rotation case. During the downstroke step, at $t=t_{1}$, the blade experiences large angles of attack ( $\alpha=\alpha_{0}+\Delta \alpha \approx 30 \mathrm{deg}$ ), which results in a large separation in the vicinity of the blade tip as well as the periodic emission of coherent vortices at the trailing edge. The separation on the suction side is progressively reduced when the blade reaches its extremum position (at the end of the downstroke step), and the flow angle at the blade leading edge decreases at the beginning of the upstroke step, at $t=t_{2}$. At the end of the downstroke step, typical flow patterns are

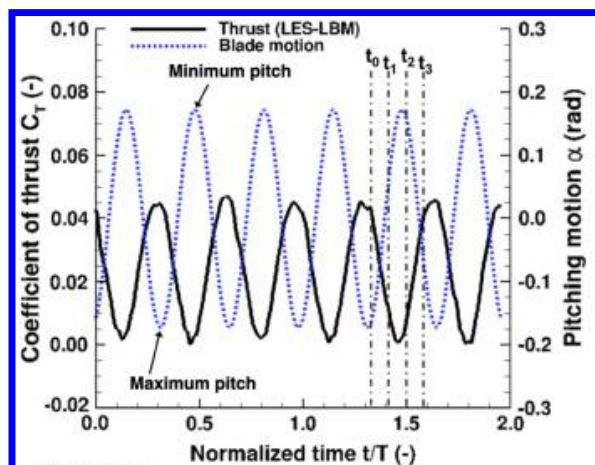

a) Pitching

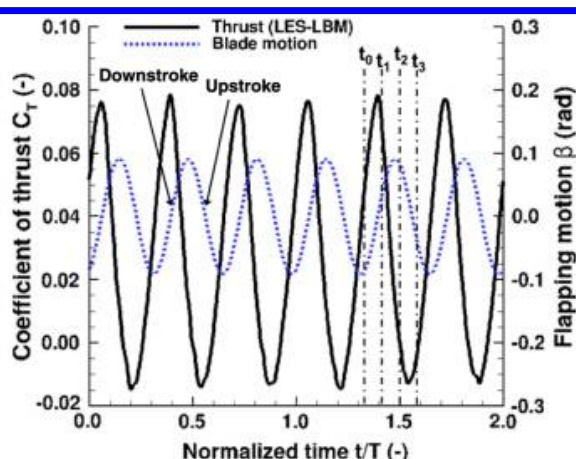

b) Flapping

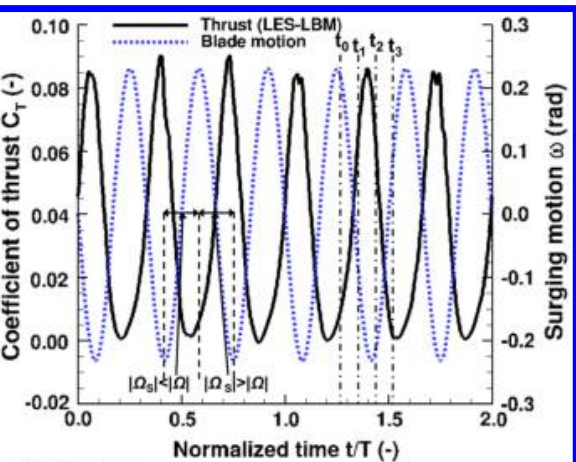

c) Surging

Fig. 9 Correlation between thrust coefficient $C_{T}(t)$ and blade motion with respect to time: a) pitching, b) flapping, and c) surging.

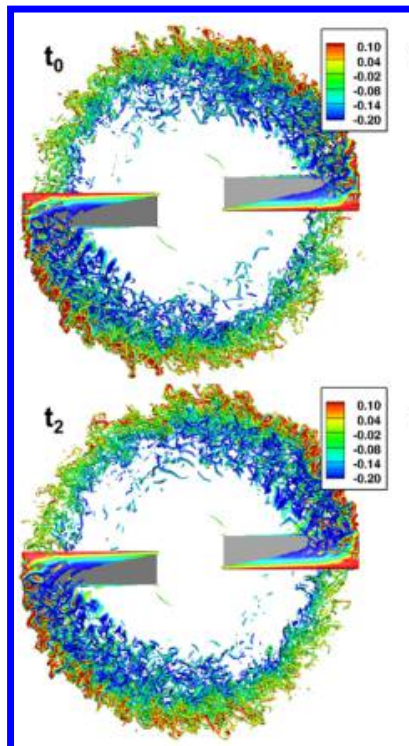

a) $Q$ criterion

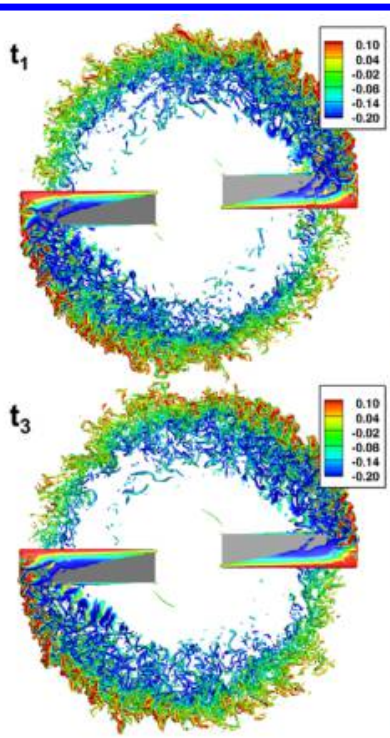

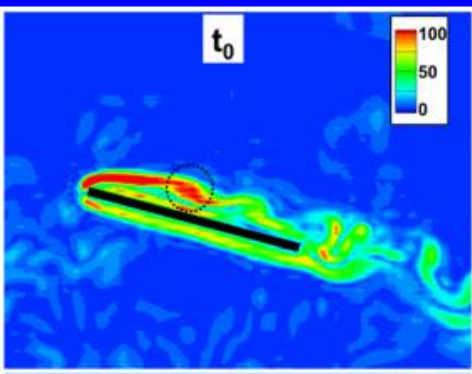

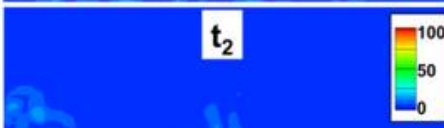

b) Vorticity

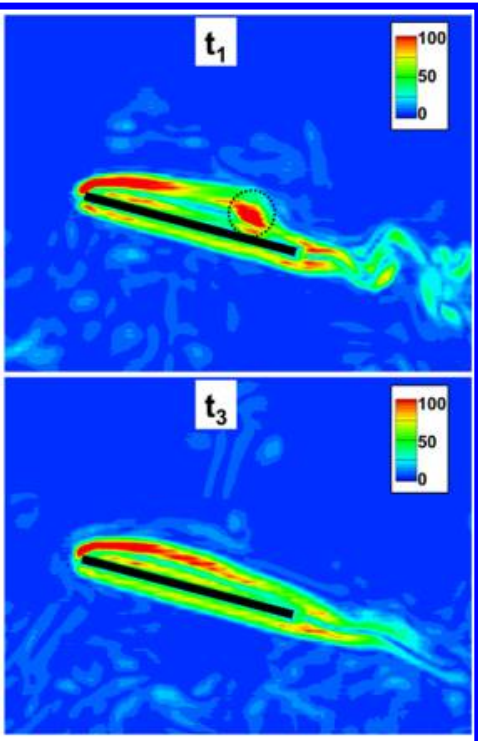

Fig. 10 Instantaneous flowfields for pure rotation case: a) isosurface of $Q$ criterion colored with normalized streamwise velocity $V_{z} /(\Omega \cdot R)$, and b) slice at $r / R=0.8$ colored with vorticity $|\nabla \times V| / \Omega$. 


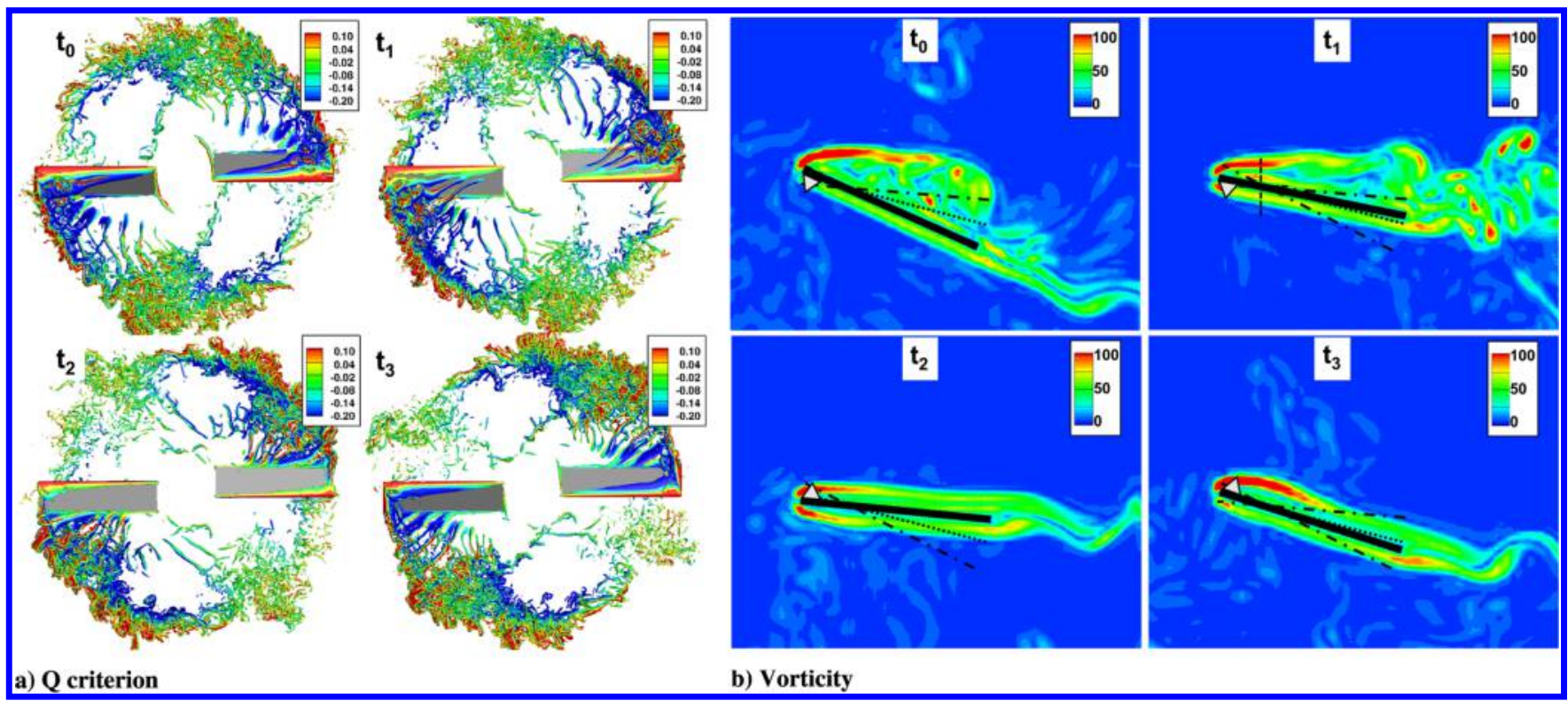

Fig. 11 Instantaneous flowfields at four different instants for pitching case: a) isosurface of $Q$ criterion colored with normalized streamwise velocity $V_{z} /(\Omega \cdot R)$, and b) slice at $r / R=0.8$ colored with vorticity $|\nabla \times V| / \Omega$.

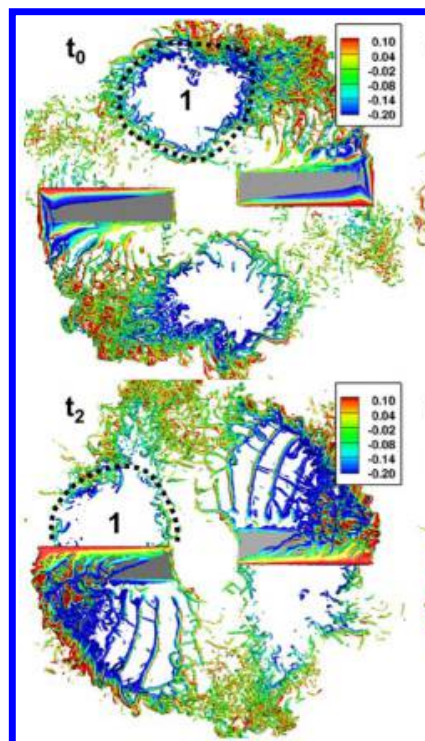

a) $Q$ criterion

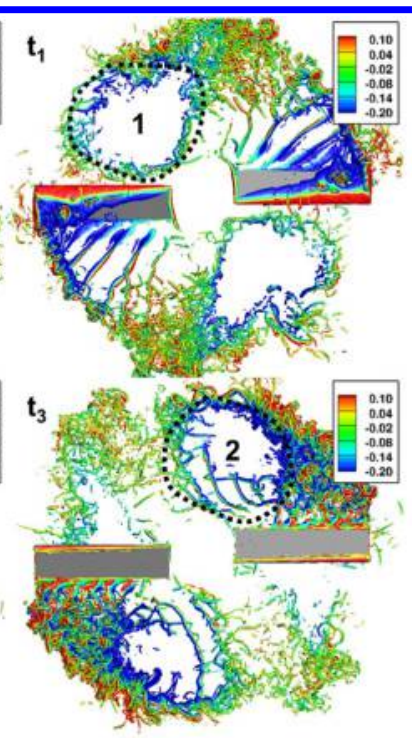

b) Vorticity
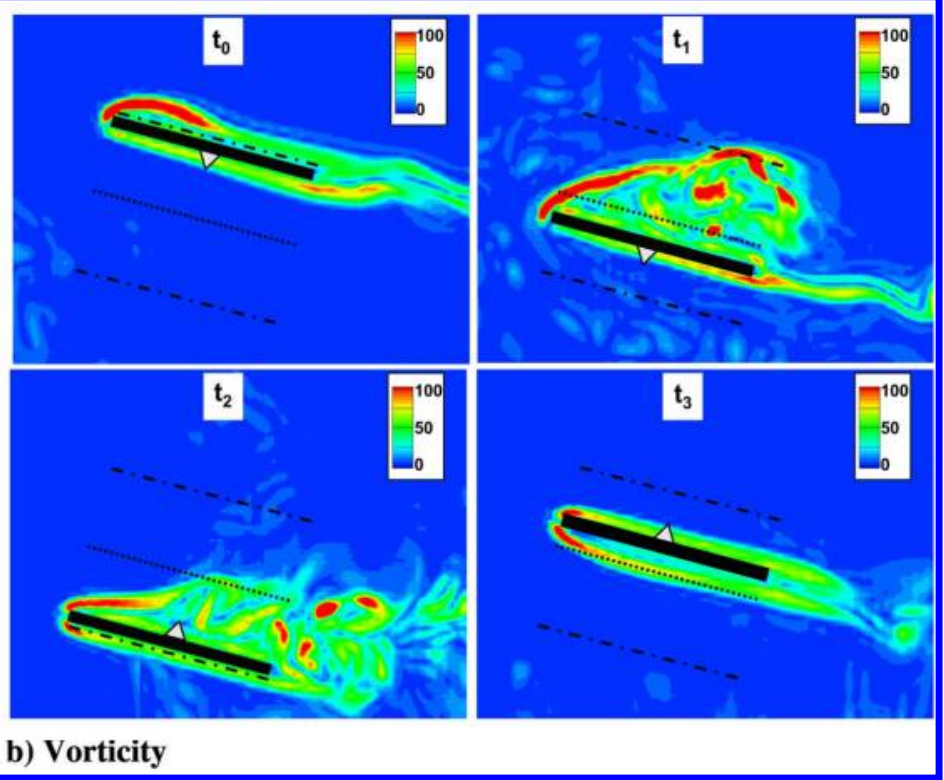

Fig. 12 Instantaneous flowfields at four different instants for flapping case: a) isosurface of $Q$ criterion colored with normalized streamwise velocity $V_{z} /(\Omega \cdot R)$, and b) slice at $r / R=0.8$ colored with vorticity $|\nabla \times V| / \Omega$.

created by the blades, which are denoted by numbers 1 and 2 in Fig. 12a; they are then convected with the flow. During the upstroke step, at $t=t_{3}$, the boundary layer on the pressure side is separated and the thrust is negative.

\section{Effect of Surging}

Instantaneous flowfields for the surging case are plotted in Fig. 13 at four different instants, describing a period of time associated to the surging motion. The arrows indicate the directions in which the blade is moving, the dashed-dotted lines indicate the two extremum positions, and the dotted lines show the mean positions. The flow $t=t_{0}$ is associated to a position close to an extremum, and so the rotational speed of the blade at this instant is close to the mean rotational speed $\Omega$. At this position, the intensity of the leading-edge vortex is of the same magnitude order as compared to the pure rotation case. However the boundary-layer separation is reduced. During the acceleration step, at $t=t_{1}$, the blade experiences a moderate increase of the angle of attack $\left(\alpha=\alpha_{0}+\Delta \alpha \approx 18 \mathrm{deg}\right)$, which results in an increase of the vorticity associated to the leadingedge vortex. The maximum thrust is achieved after $t=t_{1}$, after the blade achieves its maximum rotation speed. In the vicinity of the extremum position, close to $t=t_{2}$, a massive separation occurs at the rotor tip, and the vorticity of the leading-edge vortex starts to decrease. During the retreating blade step, at $t=t_{3}$, the flow is fully separated and the rotor thrust reaches a minimum value (close to zero). Due to its inertia, the flow is now rotating at a higher speed than the rotor, and so the leading-edge vortex, previously attached to the blade, is blown by the flow. This process is shown in Fig. 13a by following the zone denoted by the number 1 (and then denoted by the number 2 at the beginning of a new cycle).

\section{Modeling}

Based on the results of the numerical simulations, it is possible to build an analytical model to predict the effect of pitching, flapping, and surging on the thrust coefficient. The approach relies on a quasisteady approach, as was already used for flapping wing models [33]. 


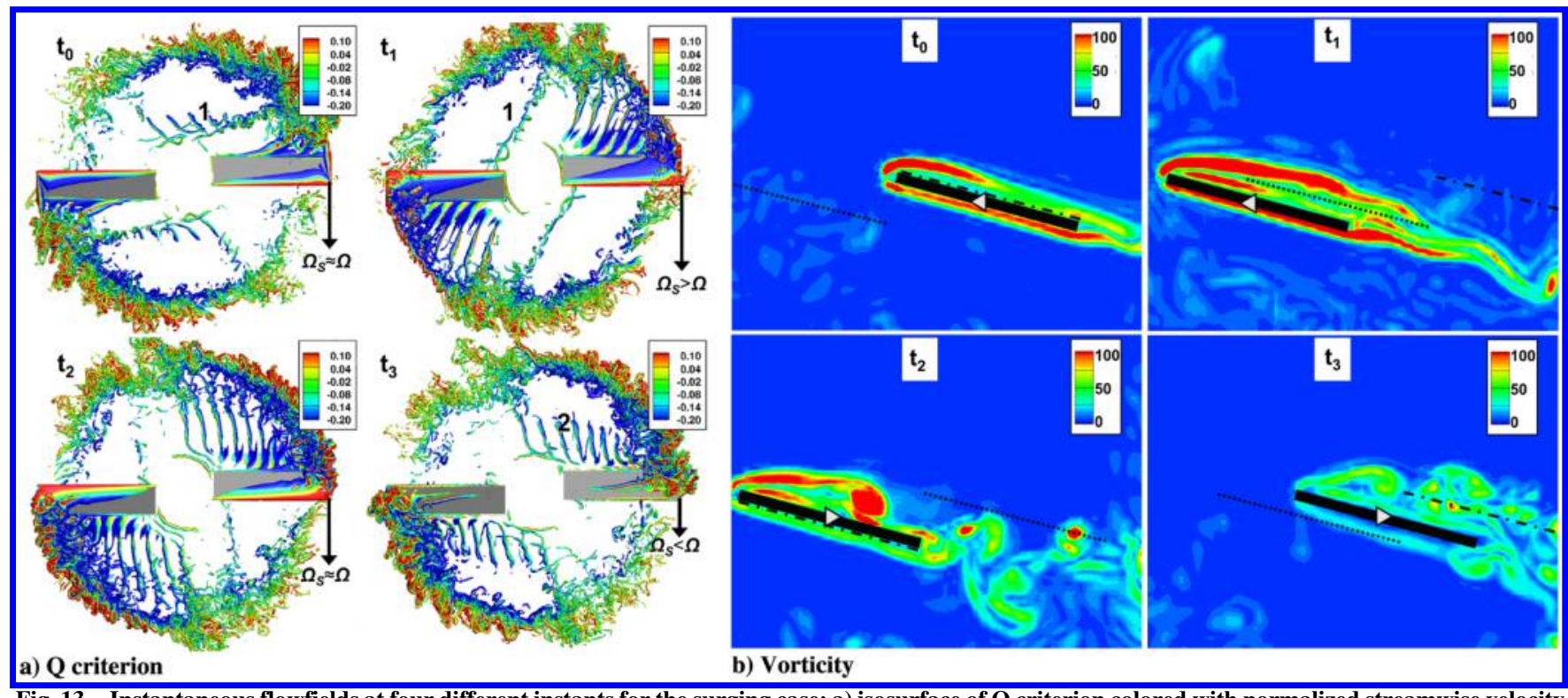

Fig. 13 Instantaneous flowfields at four different instants for the surging case: a) isosurface of $Q$ criterion colored with normalized streamwise velocity $V_{z} /(\Omega \cdot R)$, and b) slice at $r / R=0.8$ colored with vorticity $|\nabla \times V| / \Omega$.

The advantage of such an approach is that it requires only an estimation of the steady coefficient $C_{T}=f(\alpha)$, with $\alpha$ as the flow angle seen by the airfoil at the leading edge. As a first approximation, only the quadratic force terms that depend on the rotational speed at the square, such that $F=g\left(\Omega^{2}\right)$, are taken into account. Others forces like added mass effects that depend on the acceleration, such as $F=g(\Omega)$, are thus neglected (their influence is an order of magnitude lower).

\section{A. Modeling of Pitching}

The modeling of pitching effects on $C_{T}$ relies on the following decomposition:

$$
C_{T_{p}}(t)=\underbrace{C_{T}\left(\alpha_{0}+\alpha_{p}, t-\tau_{p}\right)}_{\mathrm{I}}+\underbrace{C_{T}\left(d \alpha_{p}, t-\tau_{p}\right)}_{\mathrm{II}}
$$

with $\alpha_{0}+\alpha_{p}(t)$ as the blade angle at time $t$ (quasi-steady effect, referred to as term I), $d \alpha_{p}(t)$ as the modification of the aerodynamic flow angle due to the pitching motion velocity (referred to as term II), and $\tau_{p}$ as a time lag that represents the time the thrust needs to adapt to the new flow conditions. The definition of $\alpha_{p}(t), d \alpha_{p}(t)$, and $\tau_{p}$ are, respectively, $\alpha_{p}(t)=-q_{\max } \sin \left(\omega_{m} t\right)$,

$$
d \alpha_{p}(t)=\dot{\alpha}(t)\left[x_{\mathrm{pitch}} /(\Omega \bar{r})\right]=-q_{\max } \omega_{m} \cos \left(\omega_{m} t\right)\left[x_{\mathrm{pitch}} /(\Omega \bar{r})\right]
$$

and $\tau_{p}=\bar{C} /(\Omega \cdot \bar{r})$ (averaged time needed to transport the information from the leading to the trailing edges of the airfoil). The pitching axis $x_{\text {pitch }}$ is set to $C / 4$ (corresponding to the aerodynamic center). The contribution of term I $\left[C_{T_{\mathrm{I}}}\left(\alpha_{0}+\alpha_{p}\right)\right]$ is interpolated (with a third-order polynomial) from the data reported in Fig. 6 . The contribution of term II $\left[C_{T_{\mathrm{II}}}\left(d \alpha_{p}\right)\right]$ is estimated with the thin airfoil theory [34]: in steady 2-D flow conditions, the local lift coefficient is given by $C_{l}=2 \pi d \alpha_{p}$. To partially account for 3-D effects, the local lift coefficient $C_{l}$ is integrated along the blade span, from $r$ (root) to $R$ (tip), and expressed as a thrust coefficient $C_{T}$, which is written as follows:

$$
C_{T_{\mathrm{II}}}=\frac{2}{3} \frac{C}{R}\left[1-\left(\frac{r}{R}\right)^{3}\right] d \alpha_{p}\left(t-\tau_{p}\right)
$$

For the present blade geometry, the ratios are $C / R=1 / 5$ and $r / R=1 / 5$.

This approach will produce acceptable results as long as the value of the aerodynamic angle variation $d \alpha_{p}$ is small (i.e., $d \alpha_{p}<0.3$ ), which is acceptable for the present study. Three-dimensional flow effects are also taken into account directly through the value of $C_{T}\left(\alpha_{0}+\alpha_{p}\right)$, with a good accuracy as long as the thrust coefficient is close to the mean thrust coefficient. The value of the induced velocity $V_{i}$ is also considered as constant along the disk plane, which is a questionable assumption. However, the notion of induced velocity as used here comes from the Froude theory, which is based on a onedimensional description of the flow. A more complex theory would be necessary to overcome this approximation, which is a provision for a future work.

\section{B. Modeling of Flapping}

The modeling of flapping effects on $C_{T}$ relies on a decomposition with three terms: a steady term (I) depending on the geometric blade angle $\alpha_{0}$, a term (II) related to the aerodynamic flow angle $d \alpha_{f}$ induced by the flapping motion velocity, and a term (III) depending on the effective rotation speed [the flapping is done around the hinge axis, and so a component depending on $\sin \left(\alpha_{0}\right)$ is added to the rotation speed $\Omega$ ]. Actually, the modeling of $C_{T}$ for flapping is written as follows:

$$
C_{T_{f}}(t)=[\underbrace{C_{T}\left(\alpha_{0}\right)}_{\mathrm{I}}+\underbrace{C_{T}\left(d \alpha_{f}, t-\tau_{f}\right)}_{\mathrm{II}}] \times \underbrace{\Omega_{f}^{2} / \Omega^{2}}_{\mathrm{III}}
$$

with $\tau_{f}=\bar{C} /(\Omega \cdot \bar{r})$ as the time lag, $\Omega_{f}=\Omega\left(1+\epsilon_{f}\right)$ as the effective rotation speed during flapping, $\epsilon_{f}=\left(\dot{\beta} \sin \alpha_{0}\right) / \Omega$, and $\dot{\beta}=-\left[q_{\max }\right.$. $\left.\omega_{m} \cos \left(\omega_{m} t\right)\right]$ as the flapping motion velocity. The contribution of term III $C_{T_{\mathrm{III}}}$ directly depends on the flapping axis and frequency (for example, in the case of a pure vertical flapping, the value of term III will be one). The aerodynamic angle $d \alpha_{f}$ is written, in the limit of small angles, as

$$
d \alpha_{f}(r)=\frac{\dot{\beta} \cos \alpha_{0}+\epsilon_{f}\left[V_{i} / \bar{r}\right]}{\Omega\left(1+\epsilon_{f}\right)}
$$

with $V_{i}$ as the induced velocity, which is estimated thanks to Froude theory: $V_{i}=\sqrt{T\left(\alpha_{0}\right) /\left(2 \pi \rho \cdot R^{2}\right)}$. Then, knowing the value of $d \alpha_{f}$, the contribution of term II $C_{T_{\mathrm{II}}}\left(d \alpha_{f}\right)$ is computed with Eq. (10) (as for pitching).

This model shows that flapping influences the thrust coefficient through two combined effects: an increase (respectively, decrease) of the aerodynamic angle $d \alpha$ during downstroke (respectively, upstroke) and an increase (respectively, decrease) of the effective rotation speed $\Omega_{f}$ during downstroke (respectively, upstroke). These 
Table 5 Comparison of the predicted thrust coefficient $\bar{C}_{T}$

\begin{tabular}{lcccl}
\hline \hline & Reference & Pitch & Flap & Surge \\
\hline LES-LBM & 0.0242 & 0.0251 & 0.0277 & 0.0351 \\
Model & 0.0242 & 0.0242 & 0.0280 & 0.0349 \\
$\Delta \bar{C}_{T}, \%$ & 0 & 3.5 & 1 & 0.5 \\
\hline \hline
\end{tabular}

two effects vary in phase with respect to the time, and so the effect of an increase of the aerodynamic angle is amplified by an increase of the effective rotation speed. Contrary to the pitching case, the contribution of flapping motion on $d \alpha_{f}$ is constant along the rotor span. Although the motion is symmetric with respect to the hinge axis, the combination with rotation breaks the symmetry on the flow angle, resulting in a potential creation of thrust.

For low values of $\alpha_{0}$, Eq. (12) simplifies as $d \alpha_{f} \approx \dot{\beta} / \Omega$; so, at first order, the frequency of the flapping motion is the leading parameter that explains the thrust increase. For a low ratio of $\dot{\beta} / \Omega$, the effect of flapping will be negligible on the thrust coefficient, and only term I (steady effect) will contribute to thrust. In the present case, the ratio $\dot{\beta} / \Omega$ achieves a maximum value of around 0.27 .

\section{Modeling of Surging}

As for flapping, the modeling of surging effects on $C_{T}$ is decomposed into three terms: a steady term (I) related to the geometric blade angle $\alpha_{0}$, a term (II) related to the aerodynamic flow angle $d \alpha_{f}$, and a term (III) that accounts for the modification of the effective rotation speed during surging. The thrust coefficient for surging is thus written as

$$
C_{T_{s}}(t)=[\underbrace{C_{T}\left(\alpha_{0}\right)}_{\mathrm{I}}+\underbrace{C_{T}\left(d \alpha_{s}, t-\tau_{s}\right)}_{\mathrm{II}}] \times \underbrace{\Omega_{s}^{2} / \Omega^{2}}_{\mathrm{III}}
$$

with $\tau_{s}=\bar{C} /(\Omega \cdot \bar{r})$ as the time lag, $\Omega_{s}=\Omega\left(1+\epsilon_{s}\right)$ as the effective rotation speed during surging, $\epsilon_{s}=\dot{\omega} / \Omega$, and $\dot{\omega}=-q_{\max }$. $\omega_{m} \cos \left(\omega_{m} t\right)$ as the surging motion velocity. The aerodynamic angle $d \alpha_{s}$ is written, in the limit of small angles, as

$$
d \alpha_{s}(r)=\frac{\epsilon_{s} V_{i} / \bar{r}}{\Omega\left(1+\epsilon_{s}\right)}
$$

As for the flapping case, the induced velocity $V_{i}$ is estimated with the Froude theory and $C_{T}\left(d \alpha_{s}\right)$ is computed with Eq. (10). As for flapping, the effects of both the aerodynamic angle $\overline{d \alpha}$ and the effective rotation speed $\Omega_{s}$ vary in phase, breaking the symmetry effect on the thrust coefficient. Moreover, the quadratic dependence of the thrust coefficient to the ratio $\Omega_{s} / \Omega$ will result in an increase of the mean thrust coefficient.

\section{Comparison of Models Predictions with LES-LBM}

To validate the modeling approach, a comparison of the timeaveraged thrust coefficients $\overline{C_{T}}$, as predicted with the LES-LBM and models, is shown in Table 5 . The comparison is satisfactory, especially regarding flapping $\left(\Delta C_{T}<1 \%\right)$ and surging $\left(\Delta C_{T}<0.5 \%\right)$. However, the model predicts a negligible effect of pitching on $\overline{C_{T}}$, whereas the LES-LBM shows a small gain close to $4 \%$ (1\% with URANS). The reason is that the model considers a symmetric effect of pitching velocity. Other unsteady effects, such as the influence of the leadingedge vortex circulation on the thrust, are not taken into account by the model. As a consequence, it is not possible with this model to demonstrate that pitching can have a positive effect on thrust.

A comparison of the temporal evolution of the thrust coefficient $C_{T}(t)$ is shown in Fig. 14. This comparison shows that, for these specific cases, the quasi-steady models are able to accurately reproduce the temporal evolution of thrust during pitching, flapping, and surging. During pitching (Fig. 14a), the symmetric effect of the angular velocity does not contribute directly to the mean thrust, but it reduces the effective angle seen by the profile (this effect is more important close to the root than close to the tip). This contribution [term (II) in Eq. (9)] varies with a shift in phase equal to $\pi / 2$, and so it produces the maximum effect between the two extreme positions $\left(\alpha=\alpha_{0} \pm q_{\max }\right)$. Despite its highly unsteady nature, the effect of flapping on thrust is also well reproduced by the quasi-steady model, as shown in Fig. 14b. With the chosen flapping parameters (amplitude and frequency), $7 \%$ of the mean thrust coefficient comes from the modification of the effective rotation speed [term (III) in Eq. (11)] and 9\% comes from the variation of the flow angle $d \alpha_{f}$ [term (II) in Eq. (11)]. The effect of surging on $C_{T}(t)$ is also well estimated, with only a minor shift in phase. With the chosen surging parameters (amplitude and frequency), 33\% of the thrust coefficient comes from the effective rotation speed [term (III) in Eq. (13)] and $11 \%$ comes from the variation of the aerodynamic flow angle $d \alpha_{s}$ [term (II) in Eq. (13)].

\section{Dynamic Response Motion}

The last section of this paper is dedicated to the resolution of the flow coupled with the dynamic response of the blades. The relation in Eq. (2) is solved for each blade, and so each blade is independent from the other and free to react to the aerodynamic forces. The values reported in Table $\underline{2}$ are used to investigate two cases: 1) dynamic pitching coupled with rotation, and 2) dynamic flapping coupled with rotation. In both cases, the structural damping is not considered, and so $D_{q}=0$ in Eq. (ㅁ) .

\section{A. Coupling with Pitching}

The coupled resolution is activated only after one full revolution of the rotor in order to avoid the large oscillations of the force that are associated with the first part of the transient regime. The normalized displacement and the normalized velocity displacement are plotted for the last rotation of the simulation (Fig. 15). Two conclusions are
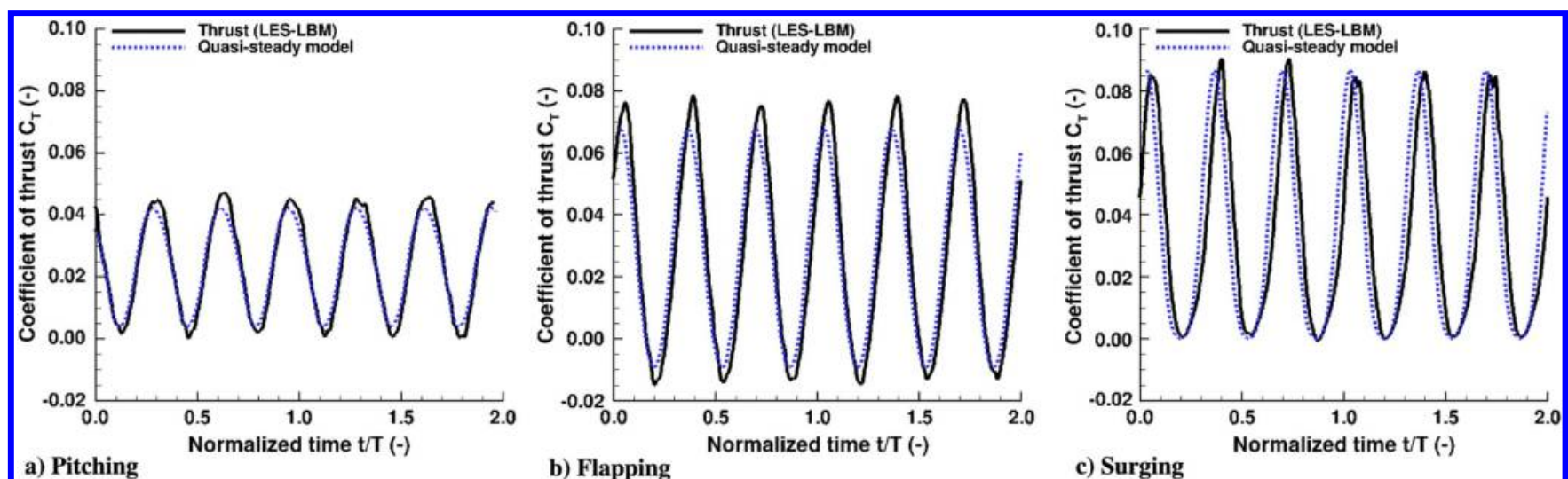

Fig. 14 Comparison of the observed thrust coefficient $C_{T}$ with a prediction model based on a quasi-steady assumption: a) pitching, b) flapping, and c) surging. 
drawn: first, the blade oscillations are periodic in time, with a frequency close to the resonance frequency of the blade, which is defined as

$$
f_{\alpha, \beta}=\frac{1}{2 \pi} \sqrt{\frac{K_{\alpha, \beta}}{I_{\alpha, \beta}}}
$$

Then, after the transient regime, the oscillations are nor damped or amplified. A residual oscillation, corresponding to less than a 0.02 deg of angle of attack, remains associated to the blade. When considering the natural frequency of the blade $\omega_{\alpha}$, the value of the reduced velocity $U^{*}$ [see Eq. ( $\underline{8}$ )] is found to be 1.33 . This means that a coupling between the flow and the blade is possible because their respective behaviors are related to the same range of frequency. An effect of less than $0.5 \%$ on the thrust and torque coefficients is observed.

The pressure coefficient defined as $C_{p}=2\left(p-p_{0}\right) /$ $\left(\rho \cdot(\Omega \cdot D / 2)^{2}\right)$ is plotted in Fig. 16 at $r / R=0.80$. As expected, the main effect is observed close to the trailing edge. On the rear part of the profile $(x / C=0.8)$, a small decrease of the flow deflection is pointed out, which is responsible for the small variation of the torque and thrust coefficients.

\section{B. Coupling with Flapping}

The simulation is now run in a coupled fashion considering the flapping mode. The normalized displacement and the normalized

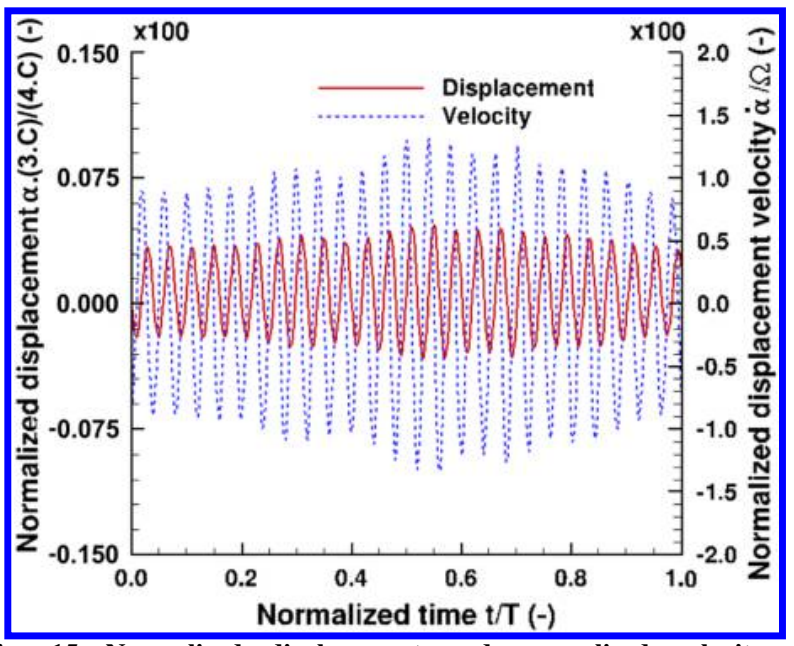

Fig. 15 Normalized displacement and normalized velocity of displacement registered at blade trailing edge during the coupled resolution of flow with dynamic pitching mode.

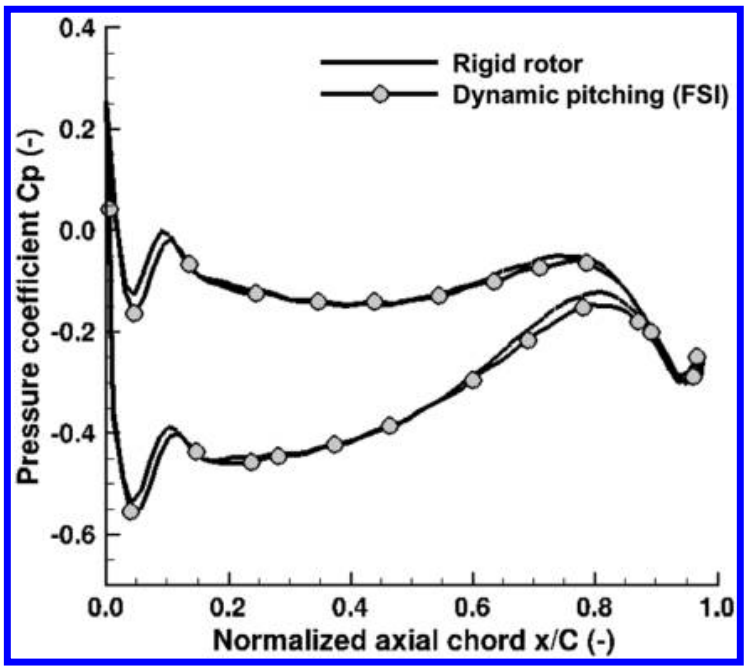

Fig. 16 Time-averaged pressure coefficient at $80 \%$ of rotor span $(r / R=0.80)$, showing influence of FSI on pressure profiles.

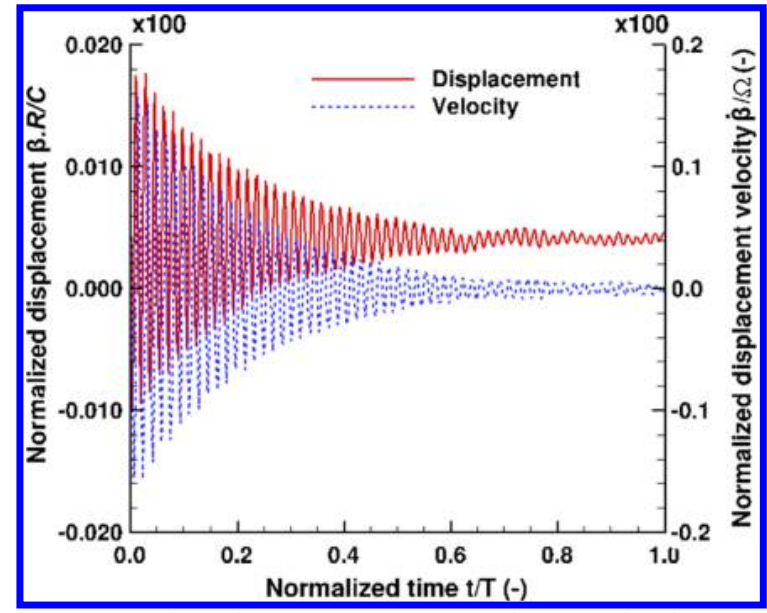

Fig. 17 Normalized displacement and normalized velocity displacement registered during coupled resolution of flow with dynamic flapping mode.

velocity displacement are plotted in Fig. 17 . Contrary to the pitching case, which shows a periodic undamped signal, the flapping motion is rapidly damped in less than half a rotation. Very small oscillations are still observed after many rotations, but they have no effect on the rotor performance. A new equilibrium position is found, very close to the uncoupled case, corresponding to an insignificant deflection corresponding to less than $0.01 \%$ of the chord in the opposite direction as compared to the induced flow. When considering the natural frequency of the blade $\omega_{\beta}$, the value of the reduced velocity $U^{*}$ [see Eq. (8)] is found to be 0.46 , which is significantly lower than one. This explains the limited interaction between the dynamic flapping and the flow.

\section{Conclusions}

Fluid-structure interaction (FSI) capabilities have been implemented in a lattice-Boltzmann method code and combined with an immersed boundary approach. The flow solver is coupled in a monolithic way, with the dynamic equation and considering a model based on an articulated plate approximation for the blades. This capability has also been used to impose a set of new kinematics based on pitching, flapping, or surging, which are superimposed to the rotation of the blades. On the one hand, the rigid nature of the material considered in this study leads to a very small influence of both dynamic pitching and flapping on the aerodynamic performance (pitching reduces the thrust by about $0.5 \%$, and flapping has a negligible effect). On the other hand, the forced motions superimposed to the rotation have a significant influence on the rotor performance: flapping and surging especially increase the thrust coefficient (up to more than $40 \%$ with surging), at the price of a penalty on the torque that partially balances the advantage on the thrust. A margin of improvement can still be expected to reduce the overcost on the torque: about $40 \%$ of the consumed power is required to impose the flapping or surging motion (the identification of more adapted kinematics to limit this overconsumption is a clear provision for a future work). An outcome of this work is also the development and validation of a set of models that predicts the effect of such complex kinematics on the thrust coefficient with a good accuracy. The research of more optimal kinematics will be largely eased by the availability of such models.

Perspectives to this work include the study of more complex motions, considering a combination of many angular velocities. Regarding FSI, future works will focus on more flexible blades (higher aspect ratio or lower stiffness).

\section{Appendix: Moments of Inertia Calculations}

Using solid cuboid formulas and Steiner's theorem [35], the moments of inertia for pitch and flap are written, respectively, as 


$$
\begin{gathered}
I_{\text {pitch }}=I_{\alpha}=\frac{1}{12} m_{S}\left(C^{2}+h^{2}\right)+m_{S}\left(\frac{C}{4}\right)^{2} \\
I_{\text {flap }}=I_{\beta} \simeq \frac{1}{12} m_{S} R_{S}^{2}+m_{S}\left(\frac{R_{S}}{2}+r\right)^{2}
\end{gathered}
$$

with $m_{S}=3.8 \times 10^{-3} \mathrm{~kg}$ as the mass of a blade, and $r$ as the distance between the main rotation axis and the hinge (i.e., the rotor radius at the root). The calculation of the stiffness $K$ relies on a beam approximation $(K=G \cdot J / L)$, with $G$ as the shear modulus and $J$ as the polar moment of the area given by

$$
J_{\text {pitch }}=J_{\alpha}=\frac{1}{12} h C\left(h^{2}+C^{2}\right)+\frac{1}{12} h C\left(\frac{C}{4}\right)^{2}
$$

and

$$
J_{\text {flap }}=J_{\beta}=\frac{h C}{12}\left(h^{2}+C^{2}\right)+R_{S} \cdot C\left(\frac{R_{S}}{2}+r\right)^{2}
$$

\section{Acknowledgments}

Numerical simulations have been performed thanks to the computing center of the Federal University of Toulouse (under projects Calcul en Midi-Pyrénées p1425 and p17014) and resources provided by Grand Équipement National de Calcul Intensif (project A0042A07178). This support is greatly acknowledged. Special thanks to Jonas Latt, from the University of Geneva and FlowKit, for his support on Palabos; and to the development team of Antares at Centre Européen de Recherche et de Formation Avancée en Calcul Scientifique for providing the postprocessing tool. The authors also thank Michael Bauerheim, from Institut Supérieur de l'Aéronautique et de l'Espace-Supaero, for very helpful discussions.

\section{References}

[1] Theodorsen, T., "General Theory of Aerodynamic Instability and the Mechanism of Flutter," Journal of the Franklin Institute, Vol. 219, No. 6, 1935, pp. 766-767.

doi:10.1016/s0016-0032(35)92022-1

[2] Greenberg, J. M., "Airfoil in Sinusoidal Motion in Pulsating Stream," NACA TN 1329, 1947.

[3] van Holten, T., "A Single Rotor Without Reaction Torque: AViolation of Newton's Laws or Feasible?" 28th European Rotorcraft Forum, Royal Aeronautical Soc., London, U.K., 2002.

[4] Fitchett, B., and Chopra, I., "A Biologically Inspired Flapping Rotor for Micro Air Vehicles," AHS International Specialists' Meeting on Unmanned Rotorcraft, American Helicopter Soc., Fairfax, VA, 2007, pp. 23-25.

[5] Guo, S., Li, D., and Wu, J., "Theoretical and Experimental Study of a Piezoelectric Flapping Wing Rotor for Micro Aerial Vehicle," Aerospace Science and Technology, Vol. 23, No. 1, 2012, pp. 429-438. doi:10.1016/j.ast.2011.10.002

[6] Wu, J., Wang, D., and Zhang, Y., "Aerodynamic Analysis of a Flapping Rotary Wing at a Low Reynolds Number," AIAA Journal, Vol. 53, No. 10,2015 , pp. 2951-2966. doi:10.2514/1.J053845

[7] Wu, J., Zhou, C., and Zhang, Y., "Aerodynamic Power Efficiency Comparison of Various Micro-Air-Vehicle Layouts in Hovering Flight," AIAA Journal, Vol. 55, No. 4, 2017, pp. 1265-1278. doi:10.2514/1.J055221

[8] Li, H., Guo, S., Zhang, Y. L., Zhou, C., and Wu, J. H., "Unsteady Aerodynamic and Optimal Kinematic Analysis of a Micro Flapping Wing Rotor," Aerospace Science and Technology, Vol. 63, April 2017, pp. $167-178$. doi:10.1016/j.ast.2016.12.025

[9] Guo, S., Li, H., Zhou, C., Zhang, Y. L., He, Y., and Wu, J. H., "Analysis and Experiment of a Bio-Inspired Flyable Micro Flapping Wing Rotor," Aerospace Science and Technology, Vol. 79, Aug. 2018, pp. 506-517. doi:10.1016/j.ast.2018.06.009

[10] Chen, L., Wu, J., Zhou, C., Hsu, S. J., and Cheng, B., "Unsteady Aerodynamics of a Pitching-Flapping-Perturbed Revolving Wing at Low Reynolds Number," Physics of Fluids, Vol. 30, No. 5, 2018,
Paper 051903

doi: $10.1063 / 1.5024925$

[11] Wang, D., Wu, J., and Zhang, Y., "Effects of Geometric Parameters on Flapping Rotary Wings at Low Reynolds Numbers," AIAA Journal, Vol. 56, No. 4, 2018, pp. 1372-1387. doi:10.2514/1.J055994

[12] Wu, J., Chen, L., Zhou, C., Hsu, S. J., and Cheng, B., "Aerodynamics of a Flapping-Perturbed Revolving Wing," AIAA Journal, Vol. 57, No. 9, 2019, pp. 3728-3743. doi:10.2514/1.j056584

[13] Jardin, T., Doue, N., Prothin, S., and Moschetta, J. M., "Numerical Analysis of Pitching-Rotor Aerodynamics," Journal of Fluids and Structures, Vol. 62, April 2016, pp. 172-186. doi:10.1016/j.jfluidstructs.2016.01.011

[14] Bos, F. M., Lentink, D., Oudheusden, B. W. V., and Bijl, H., "Influence of Wing Kinematics on Aerodynamic Performance in Hovering Insect Flight," Journal of Fluid Mechanics, Vol. 594, Jan. 2008, pp. 341-368. doi:10.1017/S0022112007009172

[15] Chimakurthi, S. K., Kang, S. K., Palacios, J., Cesnik, C. E. S., and Shyy, W., "Computational Aeroelasticity Framework for Analyzing Flapping Wing Micro Air Vehicles," AIAA Journal, Vol. 47, No. 8, 2009, pp. $1865-1878$. doi: $10.2514 / 1.38845$

[16] Dorschner, B., Chikatamarla, S. S., and Karlin, I. V., "Fluid-Structure Interaction with the Entropic Lattice Boltzmann Method," Physical Review E, Vol. 97, No. 2, 2018, Paper 023305. doi:10.1103/PhysRevE.97.023305

[17] Trapani, G., Brionnaud, R. M., and Holman, D. M., "Non-Linear FluidStructure Interaction Using a Partitioned Lattice Boltzmann-FEA Approach," 46th AIAA Fluid Dynamics Conference, AIAA AVIATION Forum, AIAA Paper 2016-3636, 2016. doi: $10.2514 / 6.2016-3636$

[18] Luo, H., Mittal, R., Zheng, X., Bielamowicz, S. A., Walsh, R. J., and Hahn, J. K., "An Immersed-Boundary Method for Flow-Structure Interaction in Biological Systems with Application to Phonation," Journal of Computational Physics, Vol. 227, No. 22, 2008, pp. 9303-9332. doi:10.1016/j.jcp.2008.05.001

[19] Gourdain, N., Singh, D., Jardin, T., and Prothin, S., "Analysis of the Turbulent Wake Generated by a Micro-Air Vehicle Hovering Near the Ground with a Lattice Boltzmann Method," Journal of American Helicopter Society, Vol. 62, No. 4, 2017, pp. 1-12. doi:10.4050/JAHS.62.042003

[20] Inamuro, T., "Lattice Boltzmann Methods for Moving Boundary," Fluid Dynamics Research, Vol. 44, No. 2, 2012, Paper 024001. doi:10.1088/0169-5983/44/2/024001

[21] Lallemand, P., and Luo, L. S., "Theory of the Lattice Boltzmann Method: Dispersion, Dissipation, Isotropy, Galilean Invariance, and Stability," Physical Review E, Vol. 61, No. 6, 2000, pp. 6546-6562. doi:10.1103/PhysRevE.61.6546

[22] D'Humieres, D., Ginzburg, I., Krafczy, M., Lallemand, P., and Luo, L. S., "Multiple Relaxation Time Lattice Boltzmann Models in ThreeDimensions," Philosophical Transactions of the Royal Society of London, Series A: Mathematical and Physical Sciences, Vol. 360, No. 1792 , 2002, pp. 437-451. doi:10.1098/rsta.2001.0955

[23] Latt, J., and Chopard, B., "Lattice Boltzmann Method with Regularized Pre-Collision Distribution Functions," Mathematics and Computers in Simulation, Vol. 72, Nos. 2-6, 2006, pp. 165-168. doi:10.1016/j.matcom.2006.05.017

[24] Malaspinas, O., Chopard, B., and Latt, J., "General Regularized Boundary Condition for Multi-Speed Lattice Boltzmann Models," Journal of Computer and Fluids, Vol. 49, No. 1, 2011, pp. 29-35. doi:10.1016/j.compfluid.2011.04.010

[25] Sagaut, P., "Toward Advanced Subgrid Models for Lattice Boltzmann Based Large-Eddy Simulation: Theoretical Formulations," Computers and Mathematics with Applications, Vol. 59, No. 7, 2010, pp. 2194 2199. doi:10.1016/j.camwa.2009.08.051

[26] Gourdain, N., Jardin, T., Serre, R., Prothin, S., and Moschetta, J.-M., "Application of a Lattice Boltzmann Method to Some Challenges Related to Micro-Air Vehicles," International Journal of Micro Air Vehicles, Vol. 10, No. 3, 2018, pp. 285-299. doi:10.1177/1756829318794174

[27] van Gerwen, D. J., and Van Holten, T., "A New Approach to Forced Flapping for the Ornicopter," 26th International Congress of the Aeronautical Sciences (ICAS), AIAA Paper 2008-8975, 2008. doi:10.2514/6.2008-8975

[28] Singh, S., and Mittal, S., "Vortex-Induced Oscillations at Low Reynolds Numbers: Hysteresis and Vortex-Shedding Modes," Journal of Fluids 
and Structures, Vol. 20, No. 8, 2005, pp. 1085-1104.

doi:10.1016/j.jfluidstructs.2005.05.011

[29] Canuto, D., and Taira, K., "Two-Dimensional Compressible Viscous Flow Around a Circular Cylinder," Journal of Fluid Mechanics, Vol. 785, Dec. 2015, pp. 349-371. doi:10.1017/jfm.2015.635

[30] Jardin, T., Prothin, S., and Garcia-Magana, C., "Aerodynamic Performance of a Hovering Microrotor in Confined Environment," Journal of American Helicopter Society, Vol. 62, No. 2, 2017, pp. 1-7. doi:10.4050/JAHS.62.022008

[31] Wojcik, C. J., and Buchholz, J. H. J., "Parameter Variation and the Leading-Edge Vortex of a Rotating Flat Plate," AIAA Journal, Vol. 52, No. 2, 2014, pp. 348-357. doi:10.2514/1.J052381

[32] Garmann, D. J., and Visbal, M. R., "Dynamics of Revolving Wings for Various Aspect Ratios," Journal of Fluids Mechanics, Vol. 748, No. 3,
2014, pp. 932-956.

doi: $10.1017 / \mathrm{jfm} .2014 .212$

[33] Kang, C. K., Aono, H., Cesnik, C., and Shyy, W., "Effects of Flexibility on the Aerodynamic Performance of Flapping Wings," Journal of Fluid Mechanics, Vol. 689, Dec. 2011, pp. 32-74. doi: $10.1017 / \mathrm{jfm} .2011 .428$

[34] Osborne, C., "Unsteady Thin-Airfoil Theory for Subsonic Flow," AIAA Journal, Vol. 11, No. 2, 1973, pp. 205-209. doi: $10.2514 / 3.6730$

[35] Abdulghany, A. R., "Generalization of Parallel Axis Theorem for Rotational Inertia," American Journal of Physics, Vol. 85, No. 10, 2017, pp. 791-795. doi: $10.1119 / 1.4994835$
S. Shin Associate Editor 